\title{
Critical resonance in the non-intersecting lattice path model
}

\author{
Richard W. Kenyon \\ CNRS UMR 8628 \\ Laboratoire de Mathématiques \\ Université Paris-Sud \\ France \\ richard.kenyon@math.u-psud.fr
}

\author{
David B. Wilson \\ Microsoft Research \\ One Microsoft Way \\ Redmond, Washington \\ U.S.A. \\ dbwilson@microsoft.com
}

\begin{abstract}
We study the phase transition in the honeycomb dimer model (equivalently, monotone non-intersecting lattice path model). At the critical point the system has a strong long-range dependence; in particular, periodic boundary conditions give rise to a "resonance" phenomenon, where the partition function and other properties of the system depend sensitively on the shape of the domain.
\end{abstract}

\section{Introduction}

We study a model of monotone non-intersecting lattice paths in $\mathbb{Z}^{2}$. Applications of this model include random surfaces $[2,23,15]$, magnetic flux lines in superconductors [32, 3], and a number of other physical phenomena [20, 11, 8], including spin-domain boundaries of the three-dimensional Ising model at zero temperature [4]. (See also [22, 13].)

Let $R_{m, n}$ be a domain consisting of the $m \times n$ rectangle in $\mathbb{Z}^{2}$ with periodic boundary conditions $\left(R_{m, n}\right.$ is a graph on a torus). On $R_{m, n}$ consider configurations consisting of collections of vertex-disjoint, monotone northeast-going lattice paths (or rather loops, since the paths are required to eventually close up, possibly after winding several times around the torus: there are no "free ends"). See Figure 1. We do not restrict the number of disjoint loops but rather give a configuration an energy $E_{b} N_{b}+E_{c} N_{c}$ where $N_{b}$ is the total number of "east" steps of the paths and $N_{c}$ is the total number of "north" steps of the paths. The Boltzmann measure $\mu$ at temperature $T$ is the probability measure assigning a configuration a probability proportional to $e^{-\left(E_{b} N_{b}+E_{c} N_{c}\right) / T}$. We study these Boltzmann measures near the critical temperature $T$, which is the temperature at which $e^{-E_{b} / T}+e^{-E_{c} / T}=1$ [14]. Letting $b=e^{-E_{b} / T}$ and $c=e^{-E_{c} / T}$, a configuration has a probability proportional to $b^{N_{b}} c^{N_{c}}$.

This process is equivalent to another well-known model, the model of dimers (weighted perfect matchings) on the honeycomb lattice $H[30,31]$. Weight the edges of the honeycomb lattice $a=1, b$, or $c$ according to their direction as in Figure 1. See Figure 1 for an illustration of the weight-preserving bijection between dimer configurations and lattice paths.

The research leading to this article was conducted in part while the first author was visiting Microsoft. 

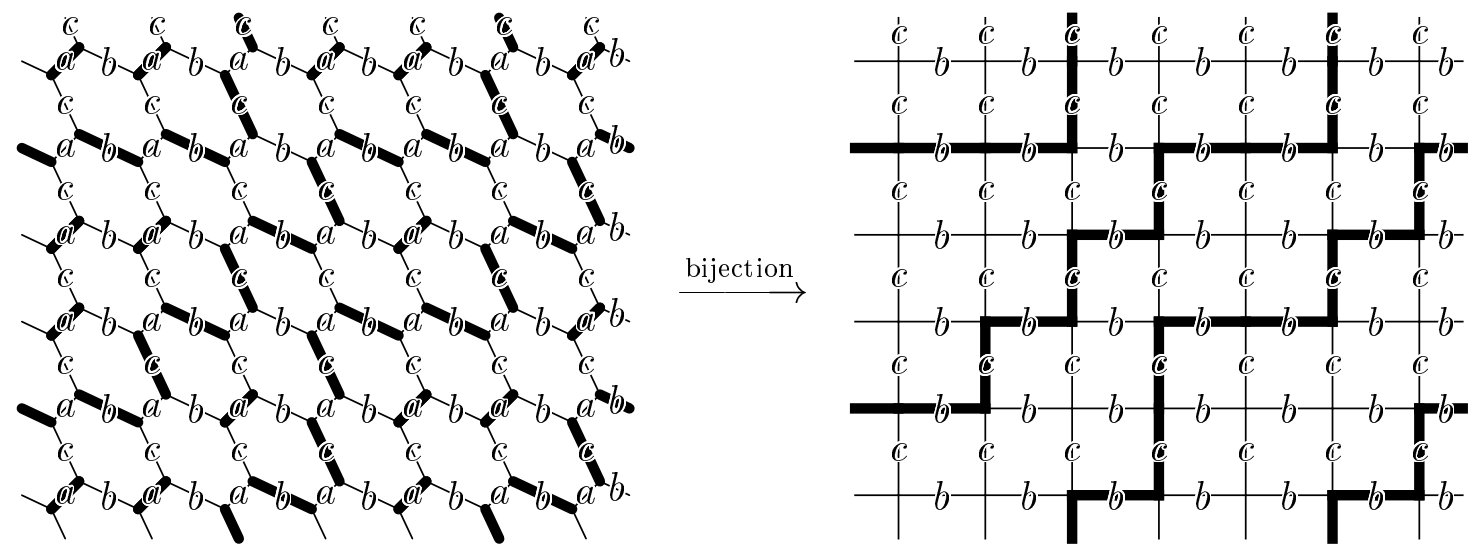

Figure 1: The classical bijection [30] between perfect matchings of the hexagonal lattice and non-intersecting north-east lattice paths, shown for a $m \times n=7 \times 6$ region. Edges of type ' $a$ ' in the hexagonal lattice $H_{m, n}$ are contracted to obtain $R_{m, n}$. The dimer configuration has weight $a^{16} b^{14} c^{12}$, as does the lattice path configuration (when $a=1$ or else one introduces a factor of $a$ for each vertex not in a lattice path).

Let $H_{m, n}$ be a finite graph which is a quotient of $H$ by the horizontal and vertical translations of length $m, n$ respectively as in Figure 1 . Let $\mu_{m, n}=\mu_{m, n}(a, b, c)$ be the Boltzmann measure on dimer configurations on the toroidal graph $H_{m, n}$. This measure assigns a dimer configuration a probability proportional to $a^{N_{a}} b^{N_{b}} c^{N_{c}}$, where there are $N_{a}$ edges of weight $a, N_{b}$ edges of weight $b$, and $N_{c}$ edges of weight $c$. The exact probability is $a^{N_{a}} b^{N_{b}} c^{N_{c}} / Z$, where the normalizing constant $Z$ is called the partition function of the system.

As $m, n \rightarrow \infty$ the measures $\mu_{m, n}(a, b, c)$ have a unique limiting Gibbs measure $\mu(a, b, c)$ $[25,6]$. The measure $\mu(a, b, c)$ is a measure on dimer configurations on $H$, and is wellunderstood for all $a, b, c[6]$. As $a, b, c$ vary the measure $\mu(a, b, c)$ undergoes a phase transition -the "solid-liquid" transition- when one of $a, b$, or $c$ is equal to the sum of the other two, for example $a=b+c$. When $a \geq b+c$ the system is frozen: with probability 1 only edges of weight $a$ are present. See Figure 2. When $a, b, c$ satisfy the strict triangle inequality (each is less than the sum of the others) dimers of all types are present in a typical configuration of $\mu(a, b, c)$. Likewise in the lattice path model, when $a \geq b+c$ there are no lattice paths. When $a, b, c$ satisfy the strict triangle inequality, the lattice paths in a configuration are dense, that is, on average they lie within a constant distance of one another.

The finite-volume measures $\mu_{m, n}(a, b, c)$ are less well understood near $a=b+c$. When $a=b+c$ the lattice paths exist but are spread out; as we shall see, the average distance between strands is on the order of the square root of the system size. The ratio $n / m$ imposes fairly rigid entropic constraints on the way these loops can join up. Surprisingly these constraints become stronger with increasing system size, so that the scaling limit has nontrivial structure.

Our main result is a computation of the partition function as a function of $a, b, c, m, n$, for parameters near $a=b+c$. The partition function $Z$ (normalized by $(\text { area })^{1 / 4}$ ) is largest when $n b /(m c)$ is rational with small numerator and denominator.

For example we have 


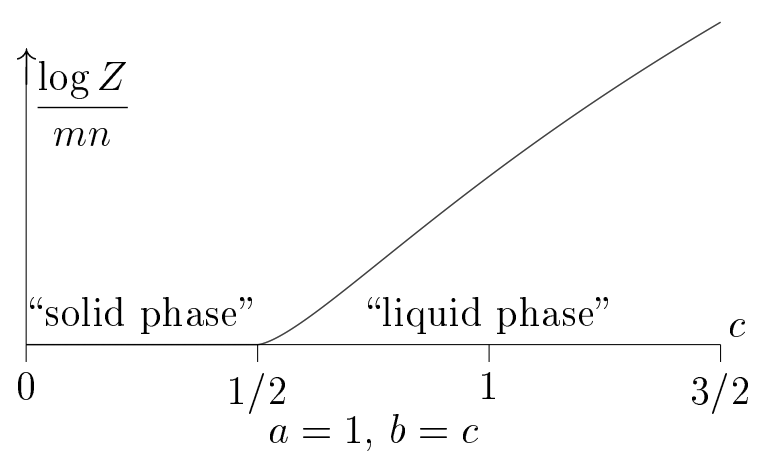

$\log Z$ along altitude of triangle

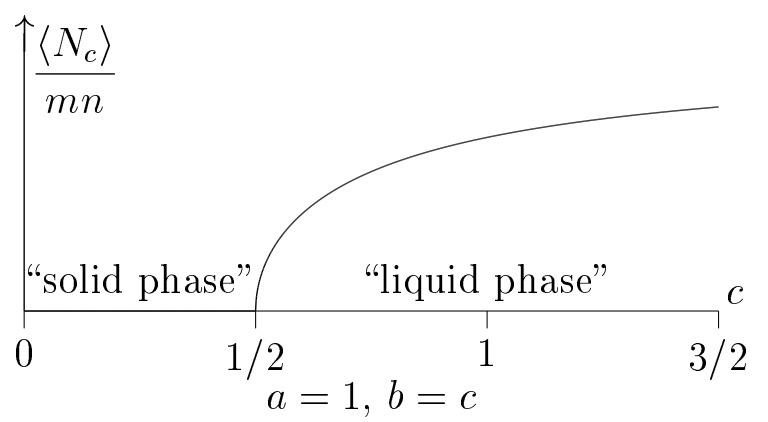

edge density along altitude of triangle
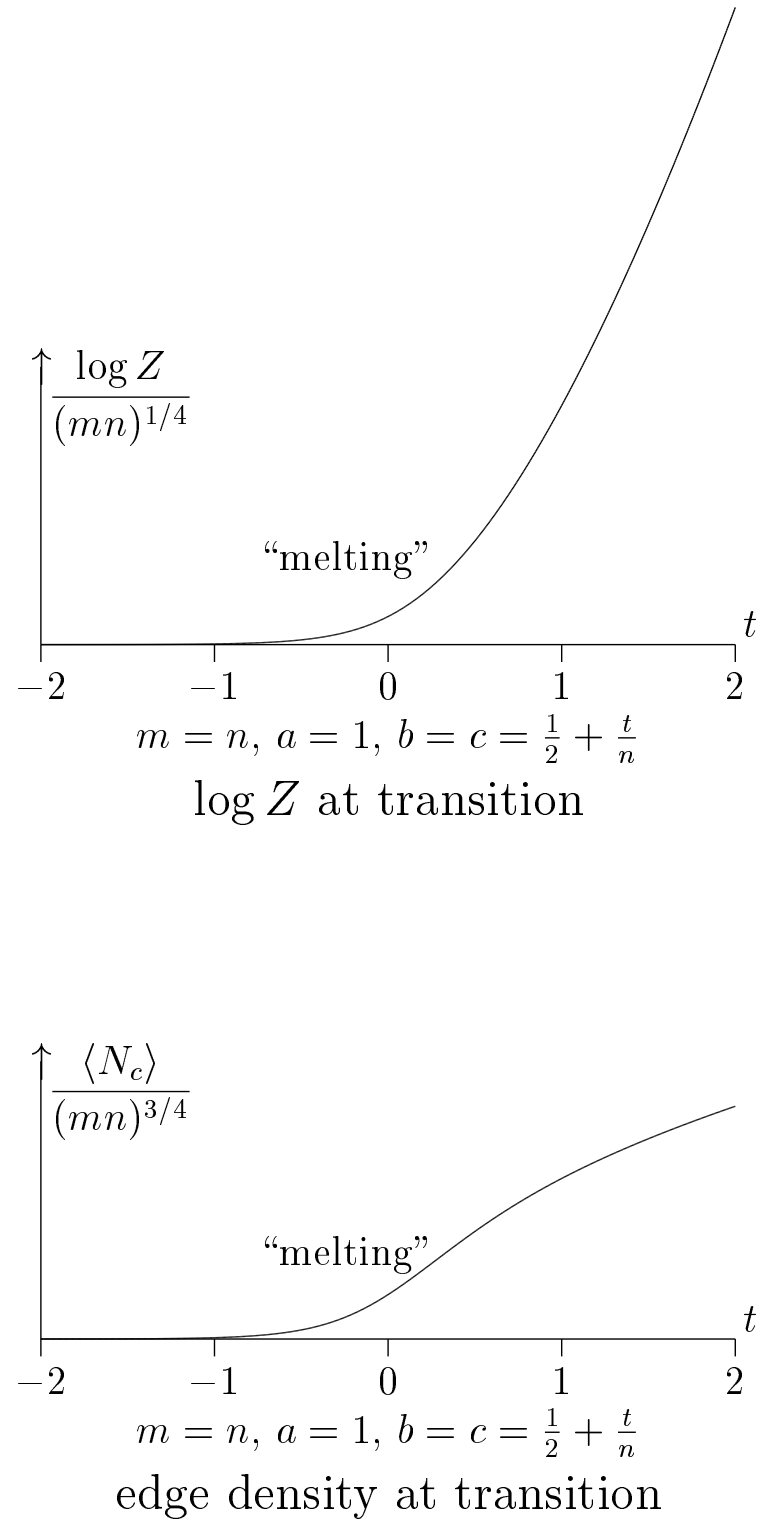

Figure 2: The partition function is shown in the upper panels, and the expected number of edges of type ' $c$ ' is shown in the lower panels. The solid and liquid phases are shown in the left panels, and the melting transition between between these phases is shown in the right panels. The formulas on the left are derived in $[14,29,6]$ and are not used here. The formulas on the right follow from Theorem 2. The melting transition depends quite sensitively on the aspect ratio of the region (see Figure 3 and Theorem 1). 
Theorem 1 When $a=1, b=c=1 / 2$ and $n / m=p / q$ in lowest terms we have

$$
\log Z=\frac{(m n)^{1 / 4} \zeta(3 / 2)\left(1-2^{-1 / 2}\right)}{2 \sqrt{\pi}(p q)^{3 / 4}}(1+o(1)) .
$$

as $m$ and $n$ tend to $\infty$ while $p$ and $q$ remain fixed (here $\zeta$ is the Riemann zeta function). Furthermore the number $N_{c}$ of 'c'-type edges tends to a Gaussian with expectation

$$
\left\langle N_{c}\right\rangle=\frac{(m n)^{3 / 4} \zeta(1 / 2)\left(1-2^{1 / 2}\right)}{2 \sqrt{\pi}(p q)^{1 / 4}}(1+o(1))
$$

and variance

$$
\sigma^{2}\left(N_{c}\right)=\frac{(m n)^{5 / 4}(p q)^{1 / 4} \zeta(-1 / 2)\left(1-2^{3 / 2}\right)}{2 \sqrt{\pi}}(1+o(1)) .
$$

(Both $\zeta(1 / 2)$ and $\zeta(-1 / 2)$ are negative.)

We see that the system greatly prefers domains of rational modulus over those with irrational modulus. Here by rational we mean, a lattice path with average slope $c / b$ will close up after winding a small number of times around the torus. In such a case the number of loops can be large, whereas in an irrational case each loop must wind many times around before closing up (unless it pays a large entropic cost).

One can think of the partition function $Z$, taken as a function of $m$ and $n$, as an indicator of rationality of $n / m$. See Figure 3 which plots $\log Z /(\text { area })^{1 / 4}$ and $\left\langle N_{c}\right\rangle /(\text { area })^{3 / 4}$ as a function of $\log (n / m)$ near area $=10^{7}$ for $a=1$ and $b=c=\frac{1}{2}$.

This is not the only interesting phenomenon in this model. For a rational domain, there is a non-trivial behavior as we vary $a, b, c$ away from the critical point. Letting $A=(c /(a-b))^{n}$, the partition function as a function of $A$ has an infinite number of nonanalyticities (in the large- $n$ limit) which correspond to abrupt changes in the winding number of curves in a typical configuration. That is, the curves "ratchet" at well-defined values as $A$ increases: see Figure 5 and $\S 5.4$. We did not prove (although we believe) that at a typical value of $A$ the curves are in a well-defined integer homology class (i.e., have well-defined winding number around the torus), and this homology class changes at discrete values of $A$. We prove only that the $\mathbb{Z}_{2}$ homology class (winding number modulo 2) changes at these well-defined points. We also show that, away from these transition points, the number of edges is a Gaussian; at the transition it is a mixture of two Gaussians, coming from a Gaussian for each homology class.

It would be very interesting to study this same model on higher-genus surfaces. On higher-genus surfaces (with translation structures having conical singularities) it would be very useful to be able to detect "rationality", in the form of bands of parallel closed geodesics: this is an important problem in billiards [26]. Moreover the ratcheting phenomenon must be significantly richer in the presence of a non-abelian fundamental group.

\section{Review of $\log Z$ and the distribution of $N_{c}$}

The partition function $Z$ is

$$
Z=\sum_{\text {configurations }} a^{N_{a}} b^{N_{b}} c^{N_{c}}
$$



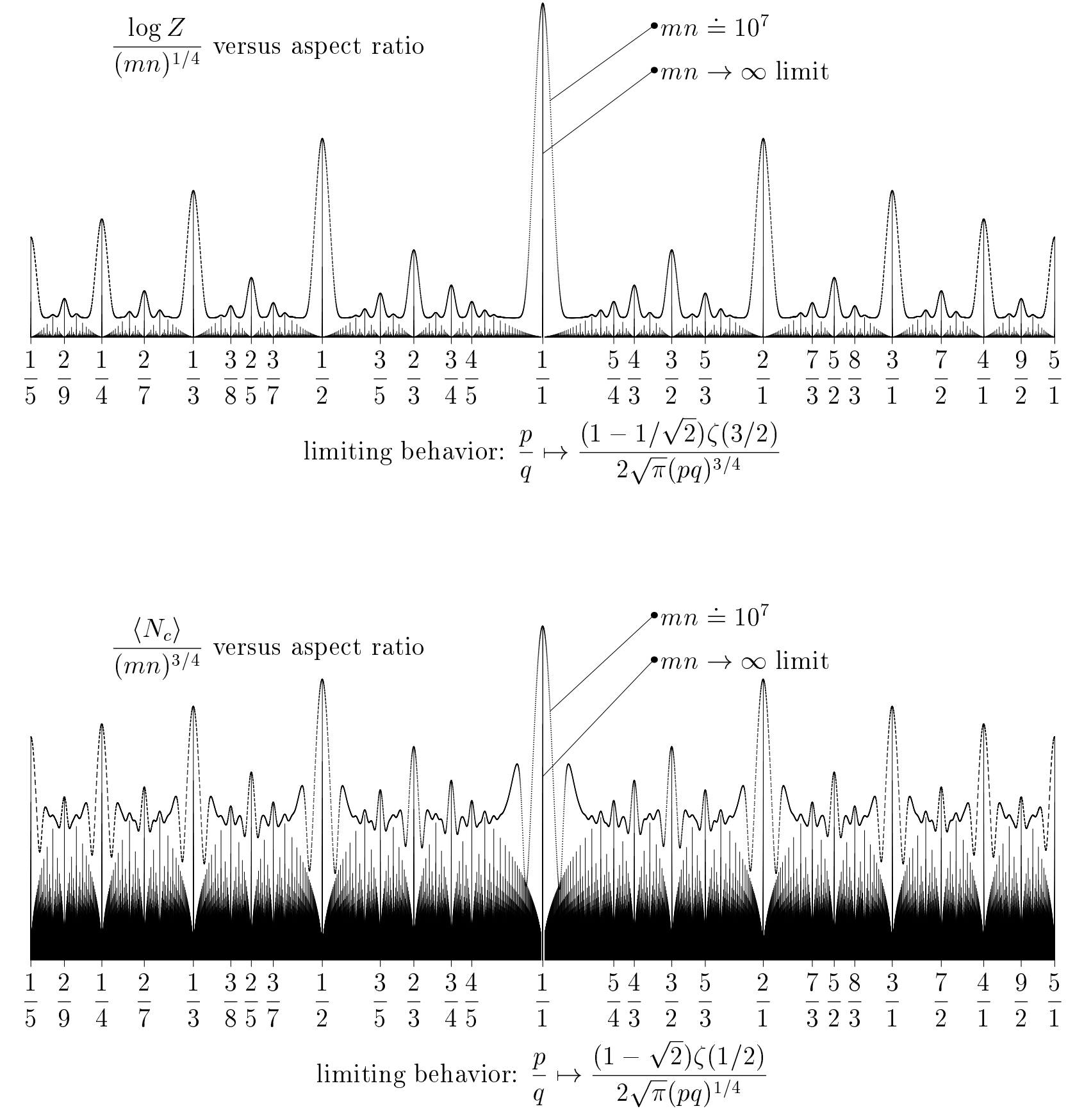

Figure 3: Resonant spikes in $\log Z$ (upper panel) and in the edge density (lower panel) for very large regions when $a=1$ and $b=c=1 / 2$. The aspect ratio is plotted on a log scale. 
It contains all the information about the distribution of the total number of edges of each type. We can view $Z$ as a polynomial in $c$, where the coefficient of $c^{N_{c}}$ is the weighted sum of configurations which contain $N_{c}$ edges of type ' $c$ '. Thus the expected number of edges of type ' $c$ ' is $\left\langle N_{c}\right\rangle=\frac{c}{Z} \frac{\partial Z}{\partial c}$. Similarly, the $\ell$ th moment of the number of edges of type ' $c$ ' is given by

$$
\left\langle N_{c}^{\ell}\right\rangle=\frac{1}{Z} \overbrace{c \frac{\partial}{\partial c} \cdots c \frac{\partial}{\partial c}}^{\ell} Z .
$$

The $\ell$ th cumulant $K_{\ell}$ is defined by

$$
K_{\ell}=\overbrace{c \frac{\partial}{\partial c} \cdots c \frac{\partial}{\partial c}}^{\ell} \log Z,
$$

which we will in effect estimate later. Here we recall several properties of cumulants [7]. Of course $\left\langle N_{c}^{1}\right\rangle=K_{1}$. The variance in the number of edges of type ' $c$ ' is $K_{2}$ :

$$
\begin{aligned}
c \frac{\partial}{\partial c} c \frac{\partial \log Z}{\partial c} & =c \frac{\partial}{\partial c} \frac{c}{Z} \frac{\partial Z}{\partial c} \\
& =\frac{c}{Z} \frac{\partial}{\partial c} c \frac{\partial Z}{\partial c}-\frac{c}{Z^{2}} \frac{\partial Z}{\partial c} c \frac{\partial Z}{\partial c} \\
& =\left\langle N_{c}^{2}\right\rangle-\left\langle N_{c}\right\rangle^{2}=\sigma^{2}\left(N_{c}\right) .
\end{aligned}
$$

Later we will use higher moments to show that the number of ' $c$ '-type edges tends to a Gaussian, and to this end we express these moments in terms of the $K_{\ell}$ 's. Since

$$
\left\langle N_{c}^{\ell+1}\right\rangle=\frac{1}{Z} \frac{c \partial}{\partial c}\left(Z\left\langle N_{c}^{\ell}\right\rangle\right)
$$

when $\left\langle N_{c}^{\ell}\right\rangle$ is expressed as a polynomial in the variables $K_{1}, \ldots, K_{\ell}$, we may calculate $\left\langle N_{c}^{\ell+1}\right\rangle$ from $\left\langle N_{c}^{\ell}\right\rangle$ by replacing each monomial $K_{i_{1}} K_{i_{2}} \cdots K_{i_{k}}$ of $\left\langle N_{c}^{\ell}\right\rangle$ with

$$
K_{1} K_{i_{1}} K_{i_{2}} \cdots K_{i_{k}}+K_{i_{1}+1} K_{i_{2}} \cdots K_{i_{k}}+K_{i_{1}} K_{i_{2}+1} \cdots K_{i_{k}}+\cdots+K_{i_{1}} K_{i_{2}} \cdots K_{i_{k}+1}
$$

Thus for example we have

$$
\begin{aligned}
& \left\langle N_{c}^{1}\right\rangle=K_{1} \\
& \left\langle N_{c}^{2}\right\rangle=K_{2}+K_{1}^{2} \\
& \left\langle N_{c}^{3}\right\rangle=K_{3}+3 K_{2} K_{1}+K_{1}^{3} \\
& \left\langle N_{c}^{4}\right\rangle=K_{4}+4 K_{3} K_{1}+3 K_{2}^{2}+6 K_{2} K_{1}^{2}+K_{1}^{4} \\
& \left\langle N_{c}^{5}\right\rangle=K_{5}+5 K_{4} K_{1}+10 K_{3} K_{2}+10 K_{3} K_{1}^{2}+15 K_{2}^{2} K_{1}+10 K_{2} K_{1}^{3}+K_{1}^{5} .
\end{aligned}
$$

These polynomials $\left\langle N_{c}^{j}\right\rangle=Y_{j}\left(K_{1}, K_{2}, \ldots\right)$ are the complete Bell polynomials [7]. We see that $\left\langle N_{c}^{\ell}\right\rangle$ contains a monomial for each partition of $\ell$, and the coefficient associated with partition with distinct part sizes $s_{1}>s_{2}>\cdots>s_{k}$ and $r_{i}$ parts of size $s_{i}$ is

$$
\frac{\ell !}{s_{1} !^{r_{1}} r_{1} ! \cdots s_{k} !{ }^{r_{k}} r_{k} !} .
$$


It will be more useful to work with moments about the mean rather than moments about the origin. Note that if we replace $Z$ with $Z^{*}=Z c^{-\mu}$, then the above derivation shows us how to express $\left\langle\left(N_{c}-\mu\right)^{\ell}\right\rangle$ in terms of the $K_{\ell}^{*}$ 's defined by

$$
K_{\ell}^{*}=\overbrace{c \frac{\partial}{\partial c} \cdots c \frac{\partial}{\partial c}}^{\ell} \log \left(Z c^{-\mu}\right)=K_{\ell}+\overbrace{c \frac{\partial}{\partial c} \cdots c \frac{\partial}{\partial c}}^{\ell} \log \left(c^{-\mu}\right) .
$$

As $K_{1}^{*}=K_{1}-\mu$ and $K_{\ell}^{*}=K_{\ell}$ for $\ell>1$, upon substituting $\mu=\left\langle N_{c}\right\rangle=K_{1}$ we see that the $\ell$ th moment of $N_{c}$ about the mean may be obtained from the above expressions for $\left\langle N_{c}^{\ell}\right\rangle$ by deleting all monomials that contain the variable $K_{1}$.

\section{Product form of the partition function}

We compute an expression for the partition function as a function of $a, b$, and $c$. We are interested in approximating $Z$ to within $1+o(1)$ multiplicative errors when $a=1, b, c \in(0,1)$ and $b+c$ is close to $a$. The interesting range is when $(c /(a-b))^{n}$ is of constant order, that is, $a-b-c=O(1 / n)$. We define $A=(c /(a-b))^{n}$ as the natural parameter measuring proximity to the critical point.

In what follows we always set $a=1$, although we keep using $a$ for notational convenience.

Recall $H_{m, n}$, the $m \times n$ hexagonal toroidal graph shown in Figure 1. By Kasteleyn [14] (see also $[27,12,24,18,19]$ for extensions and further developments), the partition function $Z=Z(a, b, c)$ for dimer coverings of $H_{m, n}$ is a sum of four expressions,

$$
Z=\frac{1}{2}\left(-Z_{00}+Z_{01}+Z_{10}+Z_{11}\right),
$$

where $Z_{\sigma \tau}$ is the determinant of a signed version of the adjacency matrix of $H_{m, n}$, and counts dimer coverings with a sign according to the homology class (in $H_{1}\left(\right.$ torus, $\left.\mathbb{Z}_{2}\right) \cong \mathbb{Z}_{2}^{2}$ ) of the corresponding system of loops, as follows. Let $N\left(\varepsilon_{\hat{x}}, \varepsilon_{\hat{y}}\right)$ denote the total weight of dimer coverings whose corresponding loops have $\varepsilon_{\hat{x}} \bmod 2$ crossings of the line $x=0$ and $\varepsilon_{\hat{y}} \bmod 2$ crossings of the line $y=0$. Each $Z_{\sigma \tau}$ is a linear combination of the $N\left(\varepsilon_{\hat{x}}, \varepsilon_{\hat{y}}\right)$ with coefficients \pm 1 as follows:

$\begin{array}{ccccc} & N(0,0) & N(1,0) & N(0,1) & N(1,1) \\ Z_{00} & +1 & -1 & -1 & -1 \\ Z_{10} & +1 & +1 & -1 & +1 \\ Z_{01} & +1 & -1 & +1 & +1 \\ Z_{11} & +1 & +1 & +1 & -1\end{array}$

Note three important facts, which follow from this table:

Proposition 1 The sum of any two of $-Z_{00}, Z_{01}, Z_{10}, Z_{11}$ has only nonnegative coefficients. The difference between any two of $-Z_{00}, Z_{01}, Z_{10}, Z_{11}$ is bounded by the sum of the other two. The difference between the coefficients of $a^{\alpha} b^{\beta} c^{\gamma}$ in any two of $-Z_{00}, Z_{01}, Z_{10}, Z_{11}$ is bounded by the sum of the coefficients of $a^{\alpha} b^{\beta} c^{\gamma}$ in the other two. 
Kasteleyn [14] evaluated the determinants $Z_{\sigma \tau}$ by multiplying eigenvalues obtained through Fourier analysis, giving

$$
\begin{aligned}
Z_{\sigma \tau} & =\prod_{\substack{(-z)^{m}=(-1)^{\sigma} \\
(-w)^{n}=(-1)^{\tau}}}[a+b z+c w] \\
& =\prod_{(-z)^{m}=(-1)^{\sigma}}\left[(a+b z)^{n}-(-1)^{\tau} c^{n}\right] \\
& =\prod_{(-z)^{m}=(-1)^{\sigma}}(a+b z)^{n} \prod_{(-z)^{m}=(-1)^{\sigma}}\left[1-(-1)^{\tau}\left(\frac{c}{a+b z}\right)^{n}\right] \\
& =\left(a^{m}-(-1)^{\sigma} b^{m}\right)^{n} \prod_{(-z)^{m}=(-1)^{\sigma}}\left[1-(-1)^{\tau}\left(\frac{c}{a+b z}\right)^{n}\right] .
\end{aligned}
$$

We can ignore the $b^{m}$ term which is exponentially smaller than $a^{m}$. When $b+c$ is close to $a$, unless $z$ is close to $-1,|a+b z|$ will be greater than $c$; in particular $|a+b z|^{n}$ is exponentially larger than $c^{n}$. So we can ignore the factors in the products for which $z$ is not close to -1 . We will expand the remaining factors near $z=-1$. As $z$ is a root of unity, let $z=z_{k}=-e^{i \theta_{k}}$, where $\theta_{k}=2 \pi k / m$ for $k \in \mathbb{Z}_{m}+\sigma / 2$. Of course $k \equiv k+m$, so when doing series expansions we can take $-m / 2<k \leq m / 2$.

Define $r_{k}, \phi_{k}$ by

$$
1 \pm\left(\frac{c}{a+b z_{k}}\right)^{n}=1 \pm\left(\frac{c}{a-b}\right)^{n} r_{k} e^{i \phi_{k}}
$$

so that

$$
r_{k}=(a-b)^{n}\left|a+b z_{k}\right|^{-n} \quad \text { and } \quad \phi_{k}=\arg \left(a+b z_{k}\right)^{-n} .
$$

We make the simplifying assumptions $b / a=\Theta(1), 1-b / a=\Theta(1)$, and $n=\Theta(m)$. Then

$$
r_{k}=\exp \left(-n \theta_{k}^{2} \frac{a b}{2(a-b)^{2}}+O\left(n \theta_{k}^{4}\right)\right)=\exp \left(-\epsilon k^{2}+O\left(k^{4} / m^{3}\right)\right)
$$

where we have defined

$$
\epsilon=\frac{2 \pi^{2} n a b}{m^{2}(a-b)^{2}}=O(1 / m)
$$

and

$$
\phi_{k}=\frac{n \theta_{k} b}{a-b}+n O\left(\theta_{k}^{3}\right)=\phi k+O\left(k^{3} / m^{2}\right)
$$

where we define

$$
\phi=\frac{2 \pi n b}{m(a-b)} .
$$

Letting $A=\left(\frac{c}{a-b}\right)^{n}$, we have

$$
Z_{\sigma \tau}=\left(a^{m}-(-1)^{\sigma} b^{m}\right)^{n} \prod_{k \in \mathbb{Z}_{m}+\sigma / 2}\left(1-(-1)^{\tau} A r_{k} e^{i \phi_{k}}\right) .
$$

In logarithmic form we can write

$$
\log Z_{\sigma \tau}=-\sum_{k \in \mathbb{Z}_{m}+\sigma / 2} \operatorname{Li}_{1}\left((-1)^{\tau} A r_{k} e^{i \phi_{k}}\right)+\underbrace{n \log \left(a^{m}-(-1)^{\sigma} b^{m}\right)}_{\text {negligible }}
$$


where the polylogarithm function $\operatorname{Li}_{\nu}$ is defined by $\operatorname{Li}_{\nu}(z)=\sum_{n=1}^{\infty} \frac{z^{n}}{n^{\nu}}$ for $|z|<1$ and by analytic continuation elsewhere (see Appendix A for background on polylogarithms). Here the second term is essentially zero, and the terms in the summation become negligible when $|k|$ is larger than $\Theta(\sqrt{m})$.

In this expression for $\log Z_{\sigma \tau}$ we need only keep track of its real part: from (6) we see that (since $b<a) Z_{\sigma \tau}$ is real and nonnegative if $A \leq 1$, and real and nonnegative if $A>1$ except for $Z_{00}$ which is strictly negative. In particular when $A>1$ the expression (3) is a sum of nonnegative terms. When $A \leq 1$ we shall see that $Z_{00}$ is negligible compared to $Z$ so its sign is irrelevant.

More generally we find, using $(7), z \frac{d}{d z} \operatorname{Li}_{\nu}(z)=\operatorname{Li}_{\nu-1}(z)$, and $(c \partial / \partial c) A=n A$, that

$$
\begin{array}{r}
\overbrace{c \frac{\partial}{\partial c} \cdots c \frac{\partial}{\partial c}}^{\ell} \log \left(Z_{\sigma \tau}\right)=-n^{\ell} \sum_{k \in \mathbb{Z}_{m}+\sigma / 2} \operatorname{Li}_{1-\ell}\left((-1)^{\tau} A r_{k} e^{i \phi_{k}}\right) \\
(+\underbrace{n \log \left(a^{m}-(-1)^{\sigma} b^{m}\right)}_{\text {negligible }} \text { if } \ell=0) .
\end{array}
$$

\section{Rational tori}

The expressions (8) are non-trivial to evaluate, mostly because they are describing behavior which depends sensitively on the parameters defining the system. In this section we compute the asymptotics of (8) for "nearly rational" domains.

We consider the toroidal hexagonal graph $H_{m, n}$ to be "nearly rational" when $\phi /(2 \pi)=$ $\frac{n b}{m(a-b)}$ is close to a simple rational $p / q$, where $p$ and $q$ are relatively prime integers. (Note that this depends not only on $m, n$ but also on $a, b, c$.) We keep $p$ and $q$ fixed as $m$ and $n$ tend to infinity, and by "close" we mean that $n b /(m c)-p / q$ is not large compared to $1 / \sqrt{q n}$. We introduce the parameter $\alpha$ to measure the closeness of $n b /(m c)$ to $p / q$ : we define $\alpha$ by

$$
\frac{\phi}{2 \pi}=\frac{p}{q}(1+\alpha W)
$$

where $W=\sqrt{q \epsilon} /(\pi p)=\Theta\left(\frac{1}{\sqrt{q n}}\right)$. The interesting range is when $\alpha$ is of constant order.

We will determine the asymptotic shape of the resonant peaks in Figure 3 as functions of $\alpha$ and $A$.

\subsection{Spokes, spirals, and clouds}

For fixed $p$ and $q$, as the area $m n$ gets large, the terms $1 \pm A r_{k} e^{i \phi_{k}} \approx 1 \pm A r_{k} e^{2 \pi i(p / q) k}$ accumulate on $q$ different spokes (or radii) of a circle with radius $A$ centered at 1 . If $\phi /(2 \pi)$ is only approximately $p / q$, then each of these spokes becomes a spiral, which spirals out from 1 when $k$ is negative and increasing, and then spirals back in towards 1 when $k$ is positive and increasing. If $\phi /(2 \pi)$ is far from a simple rational, then the terms $1 \pm A r_{k} e^{i \phi_{k}}$ form a cloud within the disk of radius $A$ centered at 1 . In our analysis for nearly rational tori, we will assume that $\phi /(2 \pi)$ is sufficiently close to a simple enough rational $p / q$ that the terms 


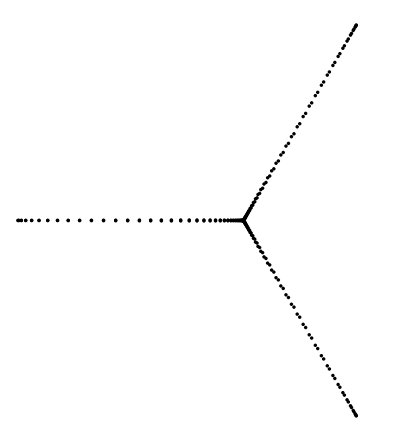

$Z_{00}$ for $60000 \times 40000$

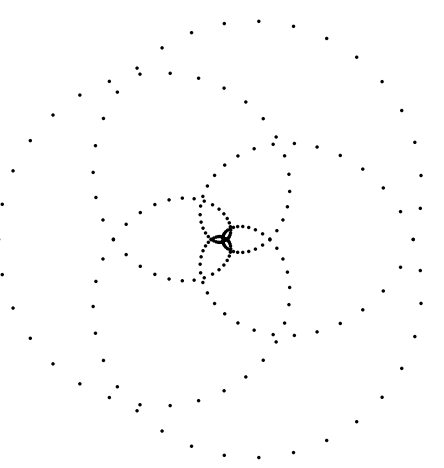

$Z_{00}$ for $60000 \times 40500$

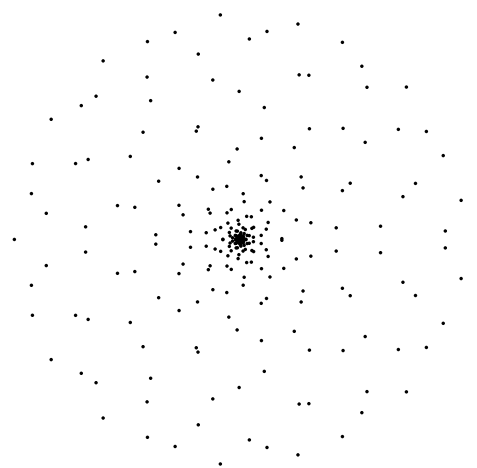

$Z_{00}$ for $75025 \times 46368$

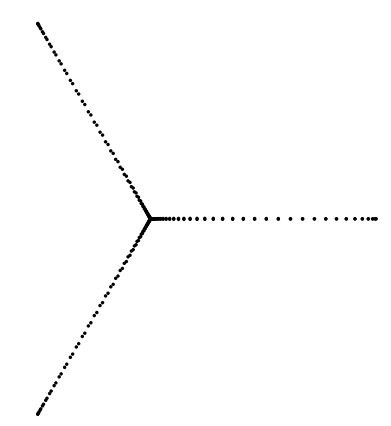

$Z_{11}$ for $60000 \times 40000$

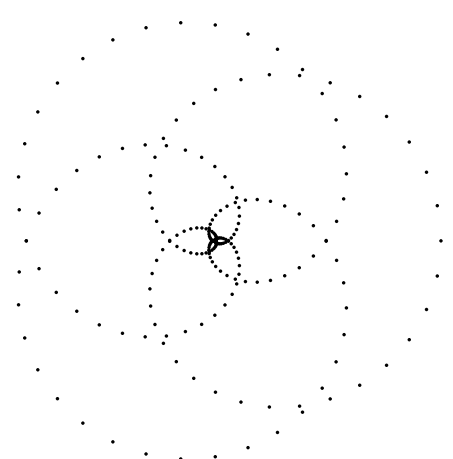

$Z_{11}$ for $60000 \times 40500$

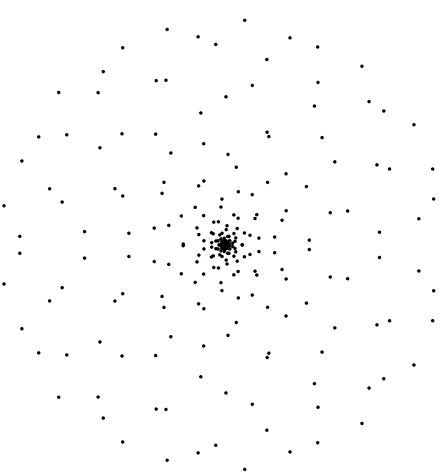

$Z_{11}$ for $75025 \times 46368$

Figure 4: The multiplicands for $Z_{00}$ and $Z_{11}$ for a domain whose aspect ratio is (a) simple rational (b) nearly simple rational (c) far from simple rational. The multiplicands are complex and accumulate towards 1.

$1 \pm A r_{k} e^{i \phi_{k}}$ form what appear to be $q$ continuous spokes or spirals (in a sense we define more precisely below). See Figure 4.

It is useful to re-express (8) to reflect the presence of the $q$ spirals. Since $\phi \approx 2 \pi \frac{p}{q}$, every $q$ th term lies on a given spiral, so we break the sum apart into $q$ different sums, one for each spiral. Of course $q$ may not evenly divide $m$. The most convenient way to re-express the summation is

$$
\sum_{k \in \mathbb{Z}_{m}+\sigma / 2} f(k)=\frac{1}{q} \sum_{j \in \mathbb{Z}_{q}+\sigma / 2} \sum_{u \in \mathbb{Z}_{m}} f(j+q u) .
$$

Since spirals are continuous objects rather than discrete sets of points, we wish to approximate $\sum_{u \in \mathbb{Z}_{m}}$ with $\int_{0}^{m} d u$. To this end we subtract $2 \pi p u$ from $\phi_{j+q u}$; then the angle $(\bmod 2 \pi)$ is unchanged for integer $u$, while for continuous $u$ it is slowly varying so we can hope that the integral approximates the sum. Thus we re-express (8) as (ignoring the negligible $O\left(n b^{m}\right)$ 
error term when $\ell=0$ )

$$
\overbrace{c \frac{\partial}{\partial c} \cdots c \frac{\partial}{\partial c}}^{\ell} \log \left(Z_{\sigma \tau}\right) \doteq-n^{\ell} \frac{1}{q} \sum_{j \in \mathbb{Z}_{q}+\sigma / 2} \sum_{u \in \mathbb{Z}_{m}} \operatorname{Li}_{1-\ell}\left((-1)^{\tau} A r_{j+q u} e^{i\left(\phi_{j+q u}-2 \pi p u\right)}\right)
$$

Later we will quantify the error introduced by approximating these sums with integrals, and show it to be insignificant so long as the points appear to line up on $q$ spirals which miss the singularity. For now we proceed with the integral approximation and simplify it:

$$
\approx-n^{\ell} \frac{1}{q} \sum_{j \in \mathbb{Z}_{q}+\sigma / 2} \int_{0}^{m} \operatorname{Li}_{1-\ell}\left((-1)^{\tau} A r_{j+q u} e^{i\left(\phi_{j+q u}-2 \pi p u\right)}\right) d u
$$

changing variables to $k=j+q u$ and using the fact that the integrand is periodic,

$$
=-n^{\ell} \frac{1}{q^{2}} \int_{0}^{q m} \sum_{j \in \mathbb{Z}_{q}+\sigma / 2} \operatorname{Li}_{1-\ell}\left((-1)^{\tau} A r_{k} e^{i\left(\phi_{k}-2 \pi k p / q\right)} e^{2 \pi i j p / q)}\right) d k
$$

using the replication formula $\frac{1}{q} \sum_{\omega^{q}=1} \operatorname{Li}_{\nu}(\omega z)=\frac{1}{q^{\nu}} \operatorname{Li}_{\nu}\left(z^{q}\right)$ and the fact that $\operatorname{gcd}(p, q)=1$,

$$
=-(q n)^{\ell} \frac{1}{q^{2}} \int_{0}^{q m} \operatorname{Li}_{1-\ell}\left((-1)^{\tau q} A^{q} r_{k}^{q} e^{i\left(\phi_{k}-2 \pi k p / q\right) q} e^{2 \pi i(\sigma / 2) p}\right) d k
$$

and again using the periodicity of $\phi_{k}$ and $r_{k}$,

$$
=-\frac{(q n)^{\ell}}{q} \int_{-m / 2}^{m / 2} \operatorname{Li}_{1-\ell}\left((-1)^{\tau q+\sigma p} A^{q} r_{k}^{q} e^{i\left(\phi_{k}-2 \pi k p / q\right) q}\right) d k
$$

which, as a function of $\sigma$ and $\tau$, only depends upon the parity of $\tau q+\sigma p$. To obtain the next formula we substitute the estimate $r_{k} e^{i \phi_{k}} \approx e^{-\epsilon(k \bmod m)^{2}} e^{i \phi(k \bmod m)}$ (where we take $k \bmod m$ to lie between $-m / 2$ and $m / 2$ ). Doing this substitution introduces an error, but we postpone the error analysis until later. Note that we can substitute this approximation for $r_{k} e^{i \phi_{k}}$ either in (10) or just prior to the integral approximation (9), since the only property of $r_{k} e^{i \phi_{k}}$ that we used in the intervening steps is that it is periodic in $k$ with period $m$.

$$
\approx-\frac{(q n)^{\ell}}{q} \int_{-m / 2}^{m / 2} \operatorname{Li}_{1-\ell}\left((-1)^{\tau q+\sigma p} A^{q} e^{-q \epsilon k^{2}} e^{i(\phi-2 \pi p / q) q k}\right) d k .
$$

We extend the range of integration to all of $\mathbb{R}$, introducing a negligible error,

$$
\approx-\frac{(q n)^{\ell}}{q} \int_{-\infty}^{\infty} \operatorname{Li}_{1-\ell}\left((-1)^{\tau q+\sigma p} A^{q} e^{-q \epsilon k^{2}} e^{i(\phi-2 \pi p / q) q k}\right) d k
$$


To measure the closeness of $\phi /(2 \pi)$ to $p / q$, we define $\alpha$ so that $\phi /(2 \pi)=p / q(1+\alpha W)$, where we define $W=\sqrt{q \epsilon} /(\pi p)$. Then $(\phi-2 \pi p / q) q k=2 \pi p \alpha W k=2 \alpha \sqrt{q \epsilon} k$. We change variables to $x=\sqrt{q \epsilon} k$ to obtain

$$
=-\frac{(q n)^{\ell}}{\sqrt{q^{3} \epsilon}} \int_{-\infty}^{\infty} \operatorname{Li}_{1-\ell}\left((-1)^{\tau q+\sigma p} A^{q} e^{2 i \alpha x-x^{2}}\right) d x .
$$

The reader may wonder about the apparent asymmetry in equation (13) (when $\ell=0$ ), e.g. why does $q$ appear but not $p$, while $Z$ is symmetrical with respect to width and height? But when $b+c \approx a \approx 1$ and $(n b) /(m c) \approx p / q$ we have

$$
\begin{gathered}
W=\frac{\sqrt{q \epsilon}}{\pi p} \approx \sqrt{\frac{2}{p m c}} \approx \sqrt{\frac{2}{q n b}} \approx \frac{\sqrt{2}}{(p q m n b c)^{1 / 4}}, \\
\frac{1}{\sqrt{q^{3} \epsilon}}=\frac{1}{\pi p q W} \approx \frac{(m n b c)^{1 / 4}}{\pi \sqrt{2}(p q)^{3 / 4}}, \\
\log A^{q}=n q \log \frac{c}{a-b} \approx(b+c-a) \frac{n q}{c} \approx(b+c-a) \frac{m p}{b}, \\
(q n)^{\ell} \approx(p q m n c / b)^{\ell / 2} .
\end{gathered}
$$

The asymmetry between $b$ and $c$ when $\ell>0$ should not be unexpected since we differentiated with respect to $c$ rather than $b$.

Referring back to the spirals in Figure $4, q$ counts the number of spirals, the parameter $A$ measures the radius of the spirals, $\sqrt{\epsilon}$ is a measure of how far apart the points are on the spiral, and $\alpha$ is a measure of the "spirality". $\alpha$ is the right parameter against which to plot the shape of the spikes, making $W$ a measure if their width. When $\alpha=0$ the spirals are spokes, when $\alpha$ gets too large (for a given $\sqrt{\epsilon}$ ) the spirals break up into a cloud, by which time the integral approximation (9) breaks down.

\subsection{Error analysis}

In the interest of simplicity, we only consider the case when $p, q, A$, and $\alpha$ are held fixed while $m \rightarrow \infty$ and $n \rightarrow \infty$. Most of the interesting behavior already shows up in this case. It is also quite interesting to ask how much these parameters can vary (e.g. can $p$ and $q$ be as large as $n^{1 / 3}$ ?), but we do not pursue that in this article.

Lemma 1 When we fix $p, q$, A, and $\alpha$ while $m \rightarrow \infty$ and $n \rightarrow \infty$, we have

$$
\frac{\sqrt{q^{3} \epsilon}}{(q n)^{\ell}} \overbrace{c \frac{\partial}{\partial c} \cdots c \frac{\partial}{\partial c}}^{\ell} \log \left(Z_{\sigma \tau}\right)=-\int_{-\infty}^{\infty} \operatorname{Li}_{1-\ell}\left((-1)^{\tau q+\sigma p} A^{q} e^{2 i \alpha x-x^{2}}\right) d x+o(1),
$$

provided that the curve $(-1)^{\tau q+\sigma p} A^{q} e^{2 i \alpha x-x^{2}}$ does not contain the point 1 . If the curve does contain 1 , then the convergence for $\ell=0$ is still valid provided that no multiplicand of $Z_{\sigma \tau}$ is closer than $e^{-o(\sqrt{n})}$ to 0 , in which case the right-hand side is merely an upper bound. 
These integrals are explicitly evaluated in $\S 5.1$.

Proof: Much of the proof has already been given in $\S 4.1$, what we have left to do is justify the approximations that we made in (9) and (11). For this error analysis we do the $r_{k} e^{i \phi_{k}} \approx e^{-\epsilon k^{2}+i \phi k}$ substitution before the integral approximation. With $k=j+q u$ and $j \in \mathbb{Z}_{q}+\sigma / 2$,

$$
\begin{aligned}
\operatorname{Li}_{1-\ell}\left((-1)^{\tau} A r_{j+q u} e^{i\left(\phi_{j+q u}-2 \pi p u\right)}\right) & =\operatorname{Li}_{1-\ell}\left((-1)^{\tau} e^{2 \pi i j p / q} A e^{i(\phi-2 \pi p / q) k-\epsilon k^{2}+O\left(k^{3} / m^{2}\right)}\right) \\
& =\operatorname{Li}_{1-\ell}\left((-1)^{\tau} e^{2 \pi i j p / q} A e^{2 i \alpha \sqrt{\epsilon / q} k-\epsilon k^{2}+O\left(k^{3} / m^{2}\right)}\right)
\end{aligned}
$$

using $\phi-2 \pi p / q=2 \pi(p / q) \alpha W=2 \alpha \sqrt{\epsilon / q}$. Next we use the fact that for integer $\ell \geq 0$, $\frac{d}{d z} \operatorname{Li}_{1-\ell}\left(e^{z}\right)=\operatorname{Li}_{-\ell}\left(e^{z}\right)$ is a rational function of $e^{z}$ with a pole at $e^{z}=1$ but which is bounded outside a neighborhood of this pole,

$$
=\operatorname{Li}_{1-\ell}\left((-1)^{\tau} e^{2 \pi i j p / q} A e^{2 i \alpha \sqrt{\epsilon / q} k-\epsilon k^{2}}\right)+O\left(k^{3} / m^{2}\right)
$$

which is valid as long as $(-1)^{\tau} e^{2 \pi i j p / q} A e^{2 i \alpha \sqrt{\epsilon / q} k-\epsilon k^{2}}$ lies outside a neighborhood of 1 , and the $O\left(k^{3} / m^{2}\right)$ error term is much smaller than the radius of this neighborhood.

It is not hard to see that the $q$ curves $(-1)^{\tau} e^{2 \pi i j p / q} A e^{2 i \alpha \sqrt{\epsilon / q} k-\epsilon k^{2}}$ are bounded away from 1 if and only if the curve $(-1)^{\tau q+\sigma p} A^{q} e^{2 i \alpha x-x^{2}}$ avoids 1 .

Adding up the errors $O\left(k^{3} / m^{2}\right)$ over the range $|k|<\Theta(\sqrt{n})$ gives $O(1)$. When $|k| \gg$ $\Theta(\sqrt{n})$, both $r_{k}$ and its approximation $e^{-\epsilon k^{2}}$ are exponentially decreasing in $|k|$, so using $\operatorname{Li}_{1-\ell}(z) \approx z$ for small $z$, and $r_{k}=e^{-\epsilon k^{2}}\left(1+O\left(k^{3} / m^{2}\right)\right)$ when $k \leq n^{2 / 3}$, we see that doing the substitution for $n^{1 / 2} \leq k \leq n^{2 / 3}$ also introduces $O(1)$ error, and that the substitution for $k \geq n^{2 / 3}$ gives $o(1)$ error. Upon multiplying by $\sqrt{q^{3} \epsilon} /(q n)^{\ell}$, all these errors become $o(1)$.

For the integral approximation (9), the integrands in (9) are continuous (except at the branch cut) as long as the curves $(-1)^{\tau} e^{2 \pi i j p / q} A e^{2 i \alpha \sqrt{\epsilon / q} k-\epsilon k^{2}}$ avoid the point 1. Moreover they converge exponentially fast to 0 when $|k| \rightarrow \infty$. Therefore they are Riemann summable and the error in converting the sums to integrals tends to zero. The error introduced by extending the range of integration to the reals is exponentially small.

What happens when a curve passes through the singularity? When $\ell=0$, the integral in expression (9) for $\log Z_{\sigma \tau}$ converges and is an upper bound for $\log Z_{\sigma \tau}$ : the Riemann sum for $\log Z_{\sigma \tau}$ converges to its integral on the complement of a small neighborhood of the singularity, and the Riemann sum near the logarithmic singularity has a negligible contribution except possibly for the point which is closest to the singularity. If the distance of the closest point to the singularity is no smaller than $e^{-o(\sqrt{n})}$, then the contribution of this point is $o(\sqrt{n})$ and so can be ignored.

\subsection{The distribution of the number of edges of type ' $c$ '}

By Lemma 1, to first order $\log Z_{\sigma \tau}$ only depends on the parity of $\tau q+\sigma p$, so we define

$$
Z_{-}=\frac{1}{2} \sum_{\substack{\sigma, \tau \\ \tau q+\sigma p \text { odd }}} Z_{\sigma \tau} \quad \text { and } \quad Z_{+}=\frac{1}{2} \sum_{\substack{\sigma, \tau \\ \tau q+\sigma p \text { even }}} \varepsilon_{\sigma \tau} Z_{\sigma \tau}
$$

where $\varepsilon_{\sigma \tau}=-1$ if $\sigma=0$ and $\tau=0$, and $\varepsilon_{\sigma \tau}=1$ otherwise. We have $Z=Z_{-}+Z_{+}$, and from Proposition 1 , both $Z_{-}$and $Z_{+}$have only nonnegative coefficients, so they can be interpreted 
as distributions. Lemma 1 shows that typically one of $Z_{-}$or $Z_{+}$is exponentially larger than the other one, so the distribution of the number of $c$ edges is governed by whichever of $Z_{-}$ or $Z_{+}$is dominant.

We use the method of moments to determine the distribution of the number $N_{c}$ of type- ' $c$ ' edges. We saw in $\S 2$ how to express the $\ell$ th moment of $N_{c}$ about its mean (call it $C_{\ell}$ ) in

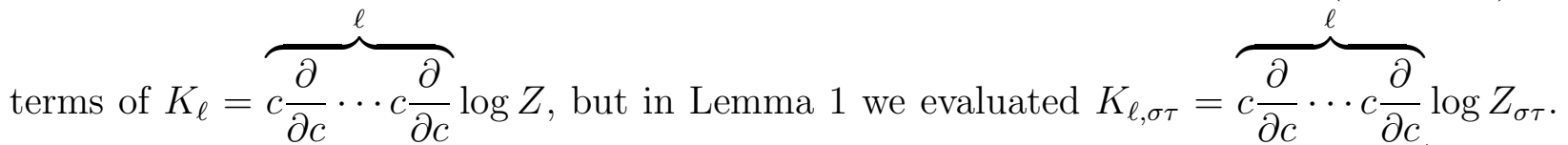
Define $C_{\ell, \sigma \tau}$ to be the same expression, except with the $K_{\ell, \sigma \tau}$ 's replacing the $K_{\ell}$ 's (see also (15) below), and similarly define the $K_{\ell, \pm}$ 's and the $C_{\ell, \pm}$ 's.

Lemma 2 Under the assumptions of Lemma 1, if the curve $(-1)^{\tau q+\sigma p} A^{q} e^{2 i \alpha x-x^{2}}$ does not contain the point 1 and $\int_{-\infty}^{\infty} \mathrm{Li}_{-1}\left((-1)^{\tau q+\sigma p} A^{q} e^{2 i \alpha x-x^{2}}\right) d x \neq 0$, then $C_{\ell, \sigma \tau} / C_{2, \sigma \tau}^{\ell / 2} \rightarrow(\ell-1) ! !$. (Here as usual $\ell ! !=\ell(\ell-2) \cdots(3)(1)$ when $\ell$ is odd and $\ell ! !=0$ when $\ell$ is even.)

Proof: From Lemma 1 we have $K_{j, \sigma \tau}=O\left((q n)^{j} / \sqrt{q^{3} \epsilon}\right)$, and since the above integral is nonzero, $C_{2, \sigma \tau}=K_{2, \sigma \tau}=\Theta\left((q n)^{2} / \sqrt{q^{3} \epsilon}\right)$. Thus each monomial in the polynomial (1) for $C_{\ell, \sigma \tau}$ has magnitude $O\left((q n)^{\ell} /\left(\sqrt{q^{3} \epsilon}\right)^{\text {degree }}\right)$. Recall that $q^{3} \epsilon \ll 1$. The monomial degree is uniquely maximized by the $K_{2, \sigma \tau}^{\ell / 2}$ term $\left(\ell\right.$ even) or the $K_{3, \sigma \tau} K_{2, \sigma \tau}^{(\ell-3) / 2}$ term $(\ell$ odd). Thus when $\ell$ is odd, $C_{\ell, \sigma \tau} / C_{2, \sigma \tau}^{\ell / 2}=O\left(\left(q^{3} \epsilon\right)^{1 / 4}\right) \rightarrow 0$, and when $\ell$ is even, $C_{\ell, \sigma \tau} / C_{2, \sigma \tau}^{\ell / 2}$ tends to the coefficient of the monomial $K_{2, \sigma \tau}^{\ell / 2}$ in $C_{\ell, \sigma \tau}$, which by $(2)$ is $(\ell-1)(\ell-3) \cdots(3)(1)$.

Thus the $C_{\ell, \sigma \tau}$ 's converge to the moments of a Gaussian, but recall that we cannot view $Z_{\sigma \tau}$ as a distribution since it may have some negative coefficients. Next we show that the moments $C_{\ell, \pm}$ of $Z_{ \pm}$(which are genuine distributions) are close to the corresponding $C_{\ell, \sigma \tau}$ 's.

Lemma 3 Suppose $\ell \in \mathbb{N}, \varsigma=(-1)^{\tau_{1} q+\sigma_{1} p}=(-1)^{\tau_{2} q+\sigma_{2} p},\left(\sigma_{1}, \tau_{1}\right) \neq\left(\sigma_{2}, \tau_{2}\right)$, and either $\varsigma=-1$, or else $\varsigma=+1$ but $A \geq 1$. Under the assumptions of Lemmas 1 and 2 , if $\mid K_{1, \sigma_{1} \tau_{1}}-$ $K_{1, \sigma_{2} \tau_{2}} \mid \ll n^{5 / 4}$ then $C_{\ell, \varsigma} / C_{2, \sigma_{1} \tau_{1}}^{\ell / 2}=(\ell-1) ! !+o(1)$.

Proof: $\quad$ Viewing $Z_{\sigma \tau}$ as a polynomial in $c, Z_{\sigma \tau}=\sum_{\imath} \gamma_{\imath, \sigma \tau} c^{\imath}$, we have

$$
C_{\ell, \sigma \tau} Z_{\sigma \tau}=\sum_{\imath} \gamma_{\imath, \sigma \tau}\left(\imath-K_{1, \sigma \tau}\right)^{\ell} c^{\imath}
$$

As $Z_{\varsigma}$ is the average of $\varepsilon_{\sigma_{1} \tau_{1}} Z_{\sigma_{1} \tau_{1}}$ and $\varepsilon_{\sigma_{2} \tau_{2}} Z_{\sigma_{2} \tau_{2}}$, we have

$$
\begin{aligned}
C_{\ell, \varsigma} Z_{\varsigma} & =\frac{1}{2} \sum_{\sigma, \tau} \varepsilon_{\sigma \tau} \sum_{\imath} \gamma_{\imath, \sigma \tau} c^{\imath}\left(\imath-K_{1, \varsigma}\right)^{\ell} \\
& =\frac{1}{2} \sum_{\sigma, \tau} \varepsilon_{\sigma \tau} \sum_{\imath} \gamma_{\imath, \sigma \tau} c^{\imath} \sum_{j=0}^{\ell}\left(\begin{array}{l}
\ell \\
j
\end{array}\right)\left(\imath-K_{1, \sigma \tau}\right)^{j}\left(K_{1, \sigma \tau}-K_{1, \varsigma}\right)^{\ell-j} \\
& =\frac{1}{2} \sum_{\sigma, \tau} \varepsilon_{\sigma \tau} \sum_{j=0}^{\ell}\left(\begin{array}{l}
\ell \\
j
\end{array}\right) C_{j, \sigma \tau} Z_{\sigma \tau}\left(K_{1, \sigma \tau}-K_{1, \varsigma}\right)^{\ell-j}
\end{aligned}
$$

Since $\varsigma=-1$ or else $\varsigma=+1$ but $A \geq 1, \varepsilon_{\sigma_{1} \tau_{1}} Z_{\sigma_{1} \tau_{1}}$ and $\varepsilon_{\sigma_{2} \tau_{2}} Z_{\sigma_{2} \tau_{2}}$ have the same sign, so $K_{1, \varsigma}$ is a convex combination of $K_{1, \sigma_{1} \tau_{1}}$ and $K_{1, \sigma_{2} \tau_{2}}$, and it too can differ by at most $\ll n^{5 / 4}=O\left(C_{2, \sigma \tau}^{1 / 2}\right)$ from them. Substituting $C_{j, \sigma \tau}=((j-1) ! !+o(1)) C_{2, \sigma \tau}^{j / 2}$ we get

$$
C_{\ell, \varsigma} Z_{\varsigma}=\frac{1}{2} \sum_{\sigma, \tau} \varepsilon_{\sigma \tau}((\ell-1) ! !+o(1)) C_{2, \sigma \tau}^{\ell / 2} Z_{\sigma \tau},
$$


so $C_{\ell, \varsigma}$ is a convex combination of $((\ell-1) ! !+o(1)) C_{2, \sigma_{1} \tau_{1}}^{\ell / 2}$ and $((\ell-1) ! !+o(1)) C_{2, \sigma_{2} \tau_{2}}^{\ell / 2}$.

Since $C_{2, \sigma_{1} \tau_{1}}=K_{2, \sigma_{1} \tau_{1}}$ and $C_{2, \sigma_{2} \tau_{2}}=K_{2, \sigma_{2} \tau_{2}}$, Lemma 1 and the hypothesis on the $K_{2}$ 's implies $C_{2, \sigma_{1} \tau_{1}}=C_{2, \sigma_{2} \tau_{2}}+O\left(n^{5 / 4}\right)$. Thus $C_{\ell, \varsigma}=((\ell-1) ! !+o(1)) C_{2, \sigma_{1} \tau_{1}}^{\ell / 2}$.

We have not computed $K_{1, \sigma \tau}$ to the precision that Lemma 3 would appear to suggest that we need, but all we really need is that the two relevant $K_{1, \sigma \tau}$ 's are quite close.

Lemma 4 Suppose $\varsigma=(-1)^{\tau_{1} q+\sigma_{1} p}=(-1)^{\tau_{2} q+\sigma_{2} p}$. Under the assumptions of Lemma 1, if

$$
-\int_{-\infty}^{\infty} \operatorname{Li}_{1}\left(-\varsigma A^{q} e^{2 i \alpha x-x^{2}}\right) d x<-\int_{-\infty}^{\infty} \operatorname{Li}_{1}\left(\varsigma A^{q} e^{2 i \alpha x-x^{2}}\right) d x
$$

then $\left|K_{1, \sigma_{1} \tau_{1}}-K_{1, \sigma_{2} \tau_{2}}\right| \leq \exp (-\Theta(\sqrt{n}))$.

Proof: For expository convenience say that the two $\sigma \tau$ 's for which $(-1)^{\tau q+\sigma p}=\varsigma$ are 01 and 10. From Lemma $1, Z_{01}$ and $Z_{10}$ dominate $-Z_{00}$ and $Z_{11}$ by a factor of $\exp (\Theta(\sqrt{n}))$, and then from Proposition $1,\left|Z_{01} / Z_{10}-1\right| \leq \exp (-\Theta(\sqrt{n}))$. Writing $Z_{\sigma \tau}$ as a polynomial in $c, Z_{\sigma \tau}=\sum_{\imath} \gamma_{\imath, \sigma \tau} c^{\imath}$, again from Proposition 1 we have

$$
\begin{aligned}
\left|\gamma_{\imath, 01}-\gamma_{\imath, 10}\right| & \leq-\gamma_{\imath, 00}+\gamma_{\imath, 11} \\
\left|\gamma_{\imath, 01} \imath c^{\imath}-\gamma_{\imath, 10} \imath c^{\imath}\right| & \leq \imath c^{\imath}\left(-\gamma_{\imath, 00}+\gamma_{\imath, 11}\right) \leq m n\left(-\gamma_{\imath, 00} c^{\imath}+\gamma_{\imath, 11} c^{\imath}\right) \\
\left|\sum_{\imath} \gamma_{\imath, 01} \imath c^{\imath}-\sum_{\imath} \gamma_{\imath, 10} \imath c^{\imath}\right| & \leq m n\left[-\sum_{\imath} \gamma_{\imath, 00} c^{\imath}+\sum_{\imath} \gamma_{\imath, 11} c^{\imath}\right] \\
\left|Z_{01} K_{1,01}-Z_{10} K_{1,10}\right| & \leq m n\left(-Z_{00}+Z_{11}\right) .
\end{aligned}
$$

As $Z_{01}$ and $Z_{10}$ are exponentially close to each other and exponentially dominate $-Z_{00}$ and $Z_{11}$, it must be that $K_{1,01}$ and $K_{1,10}$ are exponentially close.

Theorem 2 When we fix $p, q$, A, and $\alpha$ while $m \rightarrow \infty$ and $n \rightarrow \infty$, we have

$$
\log Z=\frac{(m n b c)^{1 / 4}}{\pi \sqrt{2}(p q)^{3 / 4}}\left[\max _{ \pm}-\int_{-\infty}^{\infty} \operatorname{Li}_{1}\left( \pm A^{q} e^{2 i \alpha x-x^{2}}\right) d x+o(1)\right]
$$

and with the exceptions noted below, the number $N_{c}$ of edges of type 'c' converges in distribution to a Gaussian, with mean

$$
\left\langle N_{c}\right\rangle=\frac{(m n c)^{3 / 4}}{\pi \sqrt{2}(p q b)^{1 / 4}}\left[-\int_{-\infty}^{\infty} \operatorname{Li}_{0}\left( \pm A^{q} e^{2 i \alpha x-x^{2}}\right) d x+o(1)\right],
$$

and variance

$$
\sigma^{2}\left(N_{c}\right)=\frac{(m n c)^{5 / 4}(p q)^{1 / 4}}{\pi \sqrt{2} b^{3 / 4}}\left[-\int_{-\infty}^{\infty} \operatorname{Li}_{-1}\left( \pm A^{q} e^{2 i \alpha x-x^{2}}\right) d x+o(1)\right],
$$

where the choice of \pm in (17) and (18) is the value that maximizes (16). The exceptions are

1. When both + and - maximize (16), the distribution of type- 'c' edges is a mixture of the two Gaussians defined above, provided that exceptions 2 and 3 do not also occur. (In the interest of space we omit the proof about the mixture of Gaussians, but we can supply it to the interested reader upon request.) 
2. If for the dominant choice of \pm in (16), the curve $\pm A^{q} e^{2 i \alpha x-x^{2}}$ passes through 1 , we do not say anything about the distribution of type- 'c' edges, but the formula for $\log Z$ remains valid. (We have reason to believe, but have not proved, that this scenario never occurs.)

3. In the event that the integral in (18) evaluates to 0, the formulas are still valid, but we no longer claim that the distribution is a Gaussian. (We believe that this scenario never occurs, Lemma 6 in $\S 5.2$ rules it out when $A \leq 1$.)

(See Appendix B for a review and discussion of the parameters $A$ and $\alpha$.)

Proof: Immediate from Lemma 1, Lemma 2, Lemma 3 (with the fact that Lemma 5 shows the dominant choice of \pm to be - when $A \leq 1$ ), Lemma 4, the fact that the moments are those of a Gaussian random variable, the method of moments, approximation (14) for $1 / \sqrt{q^{3} \epsilon}$, and $q n \approx \sqrt{p q m n c / b}$.

In the event that one of the curves $\pm A^{q} e^{2 i \alpha x-x^{2}}$ passes through the point 1, we still obtain (16) from Lemma 1 because each such curve has two corresponding $Z_{\sigma \tau}$ 's, at most one of which can have a multiplicand closer than $e^{-o(\sqrt{n})}$ to 0 .

Theorem 1 follows by plugging into Theorem $2 A=1$ and $\alpha=0$, using the explicit evaluation of the integrals in $\S 5.1$, and using $-\mathrm{Li}_{3 / 2}(-1)>-\mathrm{Li}_{3 / 2}(1),-\mathrm{Li}_{-1 / 2}(-1)>0$, and $\operatorname{Li}_{\nu}(-1)=\left(2^{1-\nu}-1\right) \zeta(\nu)$.

\section{$5 \quad$ Understanding the resonant spikes}

The resonant spikes, an example of which is shown in Figure 5, exhibit nontrivial behavior. We saw already that this behavior is determined by the integrals

$$
\int_{-\infty}^{\infty} \operatorname{Li}_{\nu}\left(\beta e^{2 i \alpha x-x^{2}}\right) d x
$$

with $\nu=1$ governing $\log Z, \nu=0$ governing $\left\langle N_{c}\right\rangle$, and $\nu=-1$ governing $\sigma^{2}\left(N_{c}\right)$. We start by explicitly evaluating these integrals in $\S 5.1$, and then investigate some of their properties in $\S 5.2, \S 5.3$, and $\S 5.4$. These subsections may be read in any order.

\subsection{The integral $\int_{-\infty}^{\infty} \operatorname{Li}_{\nu}\left(\beta e^{2 i \alpha x-x^{2}}\right) d x$}

We assume that $\beta \in \mathbb{C}, \alpha \in \mathbb{R}$, and $\nu \in \mathbb{C}$, though later we restrict $\nu$ to integers $\leq 1$. For convenience we assume $\alpha \geq 0$ since the integral is an even function of $\alpha$. When $|\beta|>1$ there is a branch cut $\left(\operatorname{Li}_{\nu}(z)\right.$ has a branch cut $\left.\{z \in \mathbb{R}: z \geq 1\}\right)$ that may be encountered when we vary $x$, so we need to specify which branch of the polylogarithm we are integrating over. Since the principal branch is the one for which $\operatorname{Li}_{\nu}\left(\beta e^{2 i \alpha x-x^{2}}\right) \rightarrow 0$ as $x \rightarrow \pm \infty$, we specify that the integrand is the principal branch of $\mathrm{Li}_{\nu}$, even though this may make the integrand only piecewise analytic as a function of $x$ as it ranges from $-\infty$ to $\infty$. In the event that $\alpha=0$ and $\beta$ is real and $\geq 1$, so as to ensure continuity in $\alpha$, we specify that $\beta e^{2 i \alpha x-x^{2}}$ lies above the branch cut for positive $x$ and below the cut for negative $x$. 


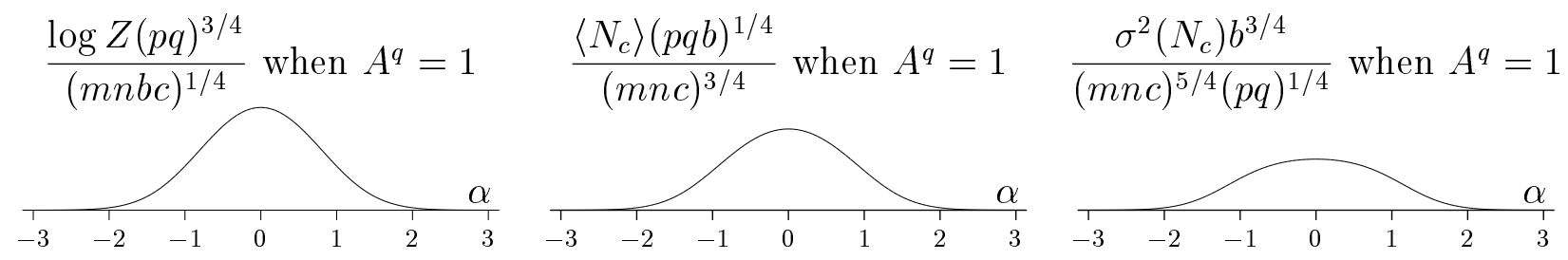

$\frac{\log Z(p q)^{3 / 4}}{(m n b c)^{1 / 4}}$ when $A^{q}=\exp (1)$

$\log Z_{-} \log Z_{+} \log Z_{-} \log Z_{+} \log Z_{-} \log Z_{+} \log Z_{+} \log Z_{-} \log Z_{+} \log Z_{-} \log Z_{+} \log Z_{-}$

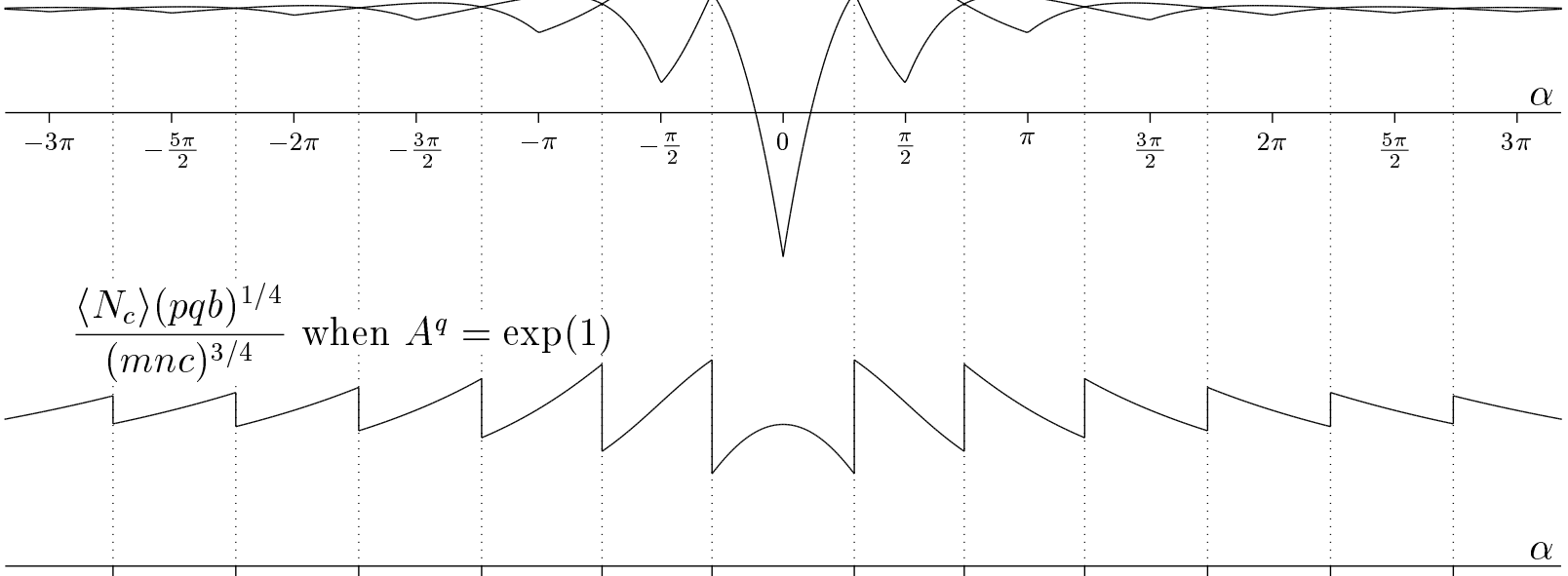

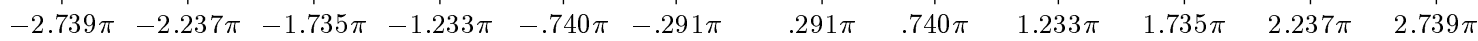

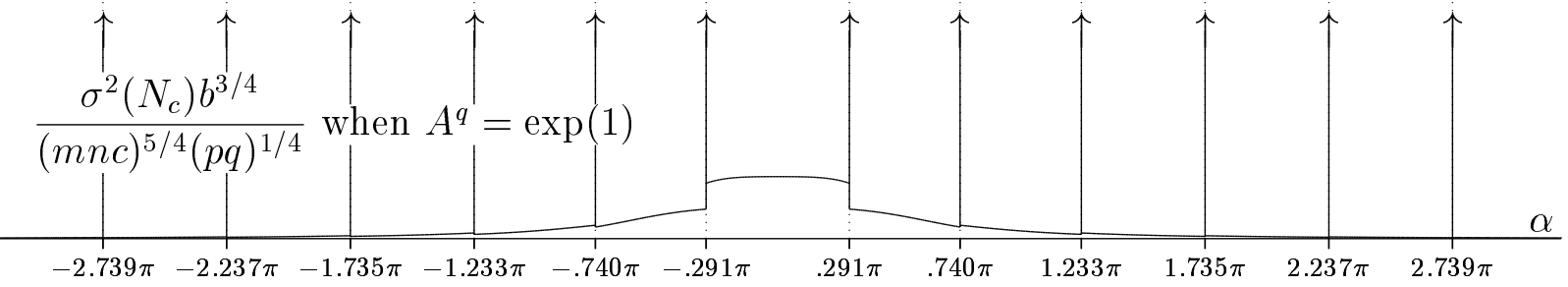

Figure 5: Anatomy of the resonant spikes. In the upper panel we show the curves for $\log Z,\left\langle N_{c}\right\rangle$, and $\sigma^{2}\left(N_{c}\right)$ as a function of $\alpha$ when $A=1$. (The parameters $A$ and $\alpha$ are reviewed in Appendix B.) When $A \leq 1, Z_{-}$is dominant, and all three curves are analytic and unimodal. The situation is quite different when $A>1$. In the next panel we show the curves for $\log Z_{+}$and $\log Z_{-}$as a function of $\alpha$ when $A^{q}=\exp (1)(\log Z$ is the $\max$ of these two curves). When $A>1$ there are singularities in $\log Z_{+}$and $\log Z_{-}$that occur when their corresponding spirals cross the singularity, that is, when $\alpha=\left(\pi / \sqrt{\log A^{q}}\right) \mathbb{Z}$ for $\log Z_{+}$and when $\alpha=\left(\pi / \sqrt{\log A^{q}}\right)(\mathbb{Z}+1 / 2)$ for $\log Z_{-}$. In the lower two panels we show the curves for $\left\langle N_{c}\right\rangle$ and $\sigma^{2}\left(N_{c}\right)$. These curves were computed using (17) and (18) as explicitly evaluated in (21) with whichever of $Z_{+}$or $Z_{-}$is significant. The "crossover points", where $\log Z_{-}$and $\log Z_{+}$alternate in significance, are in a sense a phase transition within a phase transition. At each crossover point, the curve for $\log Z$ is continuous but nonanalytic, the curve for $\left\langle N_{c}\right\rangle$ is discontinuous, and the curve for $\sigma^{2}\left(N_{c}\right)$ has a delta function. When a spiral hits the singularity, the corresponding $Z_{ \pm}$appears to be the insignificant one. The large- $\alpha$ asymptotics of the curves for $\log Z,\left\langle N_{c}\right\rangle$, and $\sigma^{2}\left(N_{c}\right)$ are given in Theorem 3 . 
We set $z=x+i y(x, y \in \mathbb{R})$ and integrate instead within the complex plane. Rather than integrate $\operatorname{Li}_{\nu}\left(\beta e^{2 i \alpha z-z^{2}}\right)$ along the real axis $\Im z=0$, it is more convenient to the integrate along the line $\Im z=\alpha$. When we deform the contour of integration and set $z=x+i \alpha$ we get

$$
\int_{-\infty}^{\infty} \operatorname{Li}_{\nu}\left(\beta e^{2 i \alpha x-x^{2}}\right) d x=\int_{-\infty}^{\infty} \operatorname{Li}_{\nu}\left(\beta e^{-\alpha^{2}} e^{-x^{2}}\right) d x+\begin{array}{r}
\text { terms from the singularities and } \\
\text { branch cuts of } \operatorname{Li}_{\nu}\left(\beta e^{2 i \alpha z-z^{2}}\right) \text { in } \\
\text { the complex } z \text {-plane. }
\end{array}
$$

In particular if $|\beta| \leq 1$ there are no singularities or branch cuts encountered when deforming the contour of integration, so there are no additional terms. We shall evaluate the main term in first. For now assume that $|\beta| e^{-\alpha^{2}}<1$ so that the series expansion of the polylogarithm is absolutely convergent. This enables us to write

$$
\begin{aligned}
\int_{-\infty}^{\infty} \operatorname{Li}_{\nu}\left(\beta e^{-\alpha^{2}} e^{-x^{2}}\right) d x & =\int_{-\infty}^{\infty} \sum_{n=1}^{\infty} \frac{\left(\beta e^{-\alpha^{2}}\right)^{n}}{n^{\nu}} e^{-n x^{2}} d x \\
& =\sum_{n=1}^{\infty} \frac{\left(\beta e^{-\alpha^{2}}\right)^{n}}{n^{\nu}} \int_{-\infty}^{\infty} e^{-n x^{2}} d x \\
& =\sum_{n=1}^{\infty} \frac{\left(\beta e^{-\alpha^{2}}\right)^{n}}{n^{\nu}} \frac{\sqrt{\pi}}{\sqrt{n}} \\
& =\sqrt{\pi} \operatorname{Li}_{\nu+1 / 2}\left(\beta e^{-\alpha^{2}}\right)
\end{aligned}
$$

The singularities of $\operatorname{Li}_{\nu}\left(\beta e^{2 i \alpha z-z^{2}}\right)$ in the complex $z$-plane occur when

$$
\begin{aligned}
\beta e^{2 i \alpha z-z^{2}} & =1 \\
2 i \alpha z-z^{2} & =-\log \beta-2 \pi i k \\
z & =i \alpha \pm i \sqrt{\alpha^{2}-\log \beta-2 \pi i k}
\end{aligned}
$$

where $k$ is an integer. Since we are moving the contour between $\Im z=0$ and $\Im z=\alpha$ (see Figure 6), the relevant singularities are of the form $i \alpha-i \sqrt{\alpha^{2}-\log \beta-2 \pi i k}$ where the principal square root is taken. (If $\arg \beta=0, \alpha^{2}<\log |\beta|$, and $k=0$ then both roots are relevant.) The branch cuts of the integrand occur where $\beta e^{2 i \alpha z-z^{2}}$ is real and $\geq 1$. With $z=x+i y$ and $x, y \in \mathbb{R}$ these are

$$
\begin{array}{rlrl}
2 \alpha x-2 x y & =-\arg \beta-2 \pi k & -2 \alpha y-x^{2}+y^{2} \geq-\log |\beta| \\
x=\frac{1}{2} \frac{2 \pi k+\arg \beta}{y-\alpha} & (y-\alpha)^{2}-x^{2} \geq \alpha^{2}-\log |\beta|
\end{array}
$$

To identify if and where the $k$ th cut intersects the line $\Im z=0$ we set $y=0$ and find that there is an intersection at

$$
-\frac{2 \pi k+\arg \beta}{2 \alpha}
$$

provided

$$
(2 \pi k+\arg \beta)^{2} \leq 4 \alpha^{2} \log |\beta|
$$




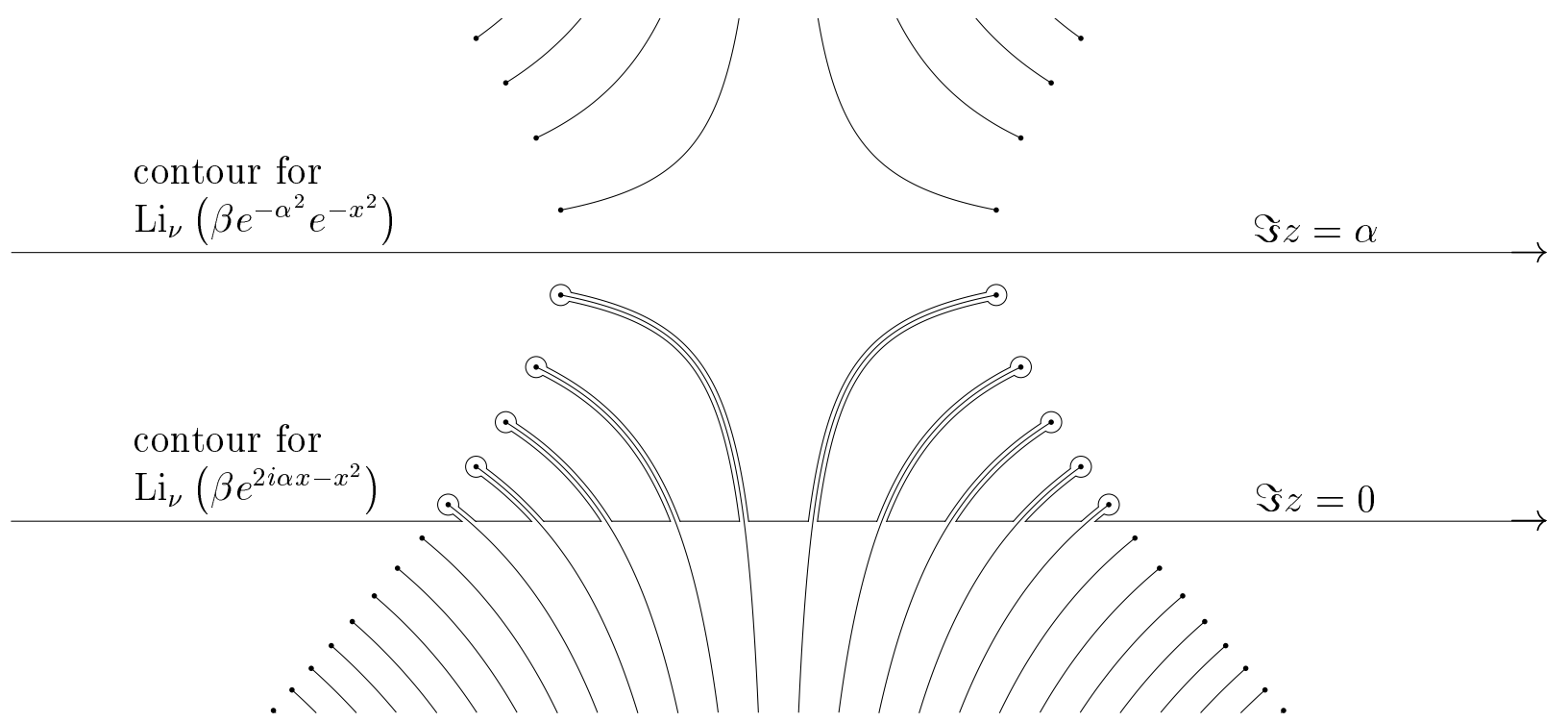

Figure 6: Singularities, branch cuts, and contours of integration for $\operatorname{Li}_{\nu}\left(\beta e^{2 i \alpha z-z^{2}}\right)$. When pushing the contour from $\Im z=\alpha$ to $\Im z=0$, the contour must deform to travel around the singularities and branch cuts, so these contribute to the integral.

For the moment suppose that $|\beta| e^{-\alpha^{2}}<1$ so that there are no singularities or branch cuts of the integrand on the line $\Im z=\alpha$. Push this contour downwards toward the line $\Im z=0$, except that when a branch cut is encountered, the contour must go around the branch cut, and travels along the cut upwards on its left side in downwards on its right side (Figure 6). The integrand increases by $(2 \pi i / \Gamma(\nu))\left(\log \left(\beta e^{2 i \alpha z-z^{2}}\right)\right)^{\nu-1}$ when going from the left side of the cut to the right side, so the net contribution of this cut to the integral is

$$
\frac{2 \pi i}{\Gamma(\nu)} \int_{C}\left(\log \left(\beta e^{2 i \alpha z-z^{2}}\right)\right)^{\nu-1} d z
$$

(plus another term from the singularity) where the contour $C$ is a branch cut travelling from the branch point to the point where the branch cut intersects the line $\Im z=0$. For the $k$ th branch cut the endpoints of integration are $i \alpha-i \sqrt{\alpha^{2}-\log \beta-2 \pi i k}$ and $-(2 \pi k+$ $\arg \beta) /(2 \alpha)$. When $\nu=1$ the singularity does not contribute and this integral is easy to evaluate and we get

$$
2 \pi i\left[-i \alpha+i \sqrt{\alpha^{2}-\log \beta-2 \pi i k}-\frac{2 \pi k+\arg \beta}{2 \alpha}\right] .
$$

Since this term gets added to the integral over $\Im z=0$, when we evaluate the integral over $\Im z=0$ we subtract this term from the integral over $\Im z=\alpha$. Thus

$$
\begin{aligned}
\int_{-\infty}^{\infty} \operatorname{Li}_{1}\left(\beta e^{2 i \alpha x-x^{2}}\right) d x= & \sqrt{\pi} \operatorname{Li}_{3 / 2}\left(\beta e^{-\alpha^{2}}\right)- \\
& 2 \pi i \sum_{\substack{k \in \mathbb{Z} \\
(2 \pi k+\arg \beta)^{2} \leq 4 \alpha^{2} \log |\beta|}}\left[-i \alpha+i \sqrt{\alpha^{2}-\log \beta-2 \pi i k}-\frac{2 \pi k+\arg \beta}{2 \alpha}\right] .
\end{aligned}
$$


There is a term in the summation for each time the spiral $\beta e^{2 i \alpha x-x^{2}}$ encloses the singularity at 1 . This formula is valid when $|\beta| e^{-\alpha^{2}}<1$, and we would like to show that it is valid (for real $\beta$ and $\alpha$ ) without this restriction. We can do this via analytic continuation in (complex) $\beta$ and $\alpha$. While both sides of equation (19) are analytic in $\alpha$ (except where the spiral $\beta e^{2 i \alpha x-x^{2}}$ crosses the singularity), neither side of (19) is analytic in $\beta$. The $\arg \beta$ terms on the right-hand side are nonanalytic, and the left-hand side is nonanalytic due to the branch cut in $\operatorname{Li}_{1}(z)$ - as $\arg \beta$ is varied, portions of the integrand cross the branch cut. To remedy this problem, we deform the branch cut as $\arg \beta$ changes, so that the cut consists of the $\operatorname{arc}\{z \in \mathbb{C}:|z|=1$ and $0 \leq \arg z \leq \arg \beta$ or $0 \geq \arg z \geq \arg \beta\}$ together with the ray $\{z \in \mathbb{C}:|z| \geq 1$ and $\arg z=\arg \beta\}$. So long as the spiral avoids the singularity, for any value of $x$, the integrand does not cross the moving branch as $\beta$ and $\alpha$ are changed. This re-interpreted integral (with the moving branch cut) is then analytic except where the spiral $\beta e^{2 i \alpha x-x^{2}}$ crosses the singularity, and the $\arg \beta$ terms on the right-hand side become constant. The $\sqrt{\pi} \mathrm{Li}_{3 / 2}\left(\beta e^{-\alpha^{2}}\right)$ term on the right-hand-side of (19) is nonanalytic when $\beta e^{-\alpha^{2}}=1$, but this nonanalyticity is cancelled by the nonanalyticity in the $k=0$ term in the summation (by (24) in Appendix A, which reviews polylogarithms). Thus the right-hand-side of the re-interpreted (19) is also analytic whenever the spiral $\beta e^{2 i \alpha x-x^{2}}$ avoids the singularity. For a given $\beta$ and $\alpha$, we may continuously decrease $|\beta|$ and increase $\alpha$ until $|\beta| e^{-\alpha^{2}}<1$ (where we already know that (19) is valid), while keeping the spiral from crossing the singularity. Thus (19) is valid for all complex $\beta$ and $\alpha$ for which the spiral avoids the singularity. As both sides of (19) are continuous, (19) is also valid when the spiral contains the singularity. We revert to the principal branch cut interpretation of the integral, restoring the $\arg \beta$ terms on the right-hand side of (19).

When $\beta$ is real, upon summing over $k$, the terms $(2 \pi k+\arg \beta) /(2 \alpha)$ cancel: when $\beta<0$ the $k$ th term cancels the $(-1-k)$ th term, and when $\beta>0$ the $k$ th term cancels the $-k$ th term, while the 0th term does not contribute. For real $\beta$ we get

$$
\int_{-\infty}^{\infty} \operatorname{Li}_{1}\left(\beta e^{2 i \alpha x-x^{2}}\right) d x=\sqrt{\pi} \operatorname{Li}_{3 / 2}\left(\beta e^{-\alpha^{2}}\right)-2 \pi \sum_{\substack{k \in \mathbb{Z} \\(2 \pi k+\arg \beta)^{2} \leq 4 \alpha^{2} \log |\beta|}}\left(\alpha-\sqrt{\alpha^{2}-\log \beta-2 \pi i k}\right) .
$$

When $\beta e^{-\alpha^{2}}>1$ the $k=0$ "branch-cut term" exactly cancels the imaginary part of the $\mathrm{Li}_{3 / 2}$ term. Applying $\beta \frac{\partial}{\partial \beta}$ multiple times to (20) we find for real $\beta$ and nonpositive integer $\nu$

$$
\int_{-\infty}^{\infty} \operatorname{Li}_{\nu}\left(\beta e^{2 i \alpha x-x^{2}}\right) d x=\sqrt{\pi} \operatorname{Li}_{\nu+1 / 2}\left(\beta e^{-\alpha^{2}}\right)-\sqrt{\pi} \Gamma\left(\frac{1}{2}-\nu\right) \sum_{\substack{k \in \mathbb{Z} \\(2 \pi k+\arg \beta)^{2} \leq 4 \alpha^{2} \log |\beta|}}\left[\alpha^{2}-\log \beta+2 \pi i k\right]^{-1 / 2+\nu} .
$$

\subsection{Inequalities}

To use Theorem 2 we need to know which of $Z_{ \pm}$is significant. We already saw that they can alternate in significance (as $\alpha$ is varied) when $A>1$. We claim that when $A \leq 1$, or else $A>1$ but $\alpha=0$, that $Z_{-}$is the significant one.

Lemma 5 If $0<\beta \leq 1$, or else $\beta>1$ but $\alpha=0$, then

$$
-\int_{-\infty}^{\infty} \operatorname{Li}_{1}\left(\beta e^{2 i \alpha x-x^{2}}\right) d x<-\int_{-\infty}^{\infty} \operatorname{Li}_{1}\left(-\beta e^{2 i \alpha x-x^{2}}\right) d x .
$$


Proof: Under either of the hypotheses, equation (20) simplifies to

$$
\int_{-\infty}^{\infty} \operatorname{Li}_{1}\left( \pm \beta e^{2 i \alpha x-x^{2}}\right) d x=\sqrt{\pi} \operatorname{Li}_{3 / 2}\left( \pm \beta e^{-\alpha^{2}}\right)=\int_{-\infty}^{\infty} \operatorname{Li}_{1}\left( \pm \beta e^{-\alpha^{2}-x^{2}}\right) d x
$$

but

$$
-\Re \operatorname{Li}_{1}\left(\beta e^{-\alpha^{2}-x^{2}}\right)=\Re \log \left(1-\beta e^{-\alpha^{2}-x^{2}}\right)<\Re \log \left(1+\beta e^{-\alpha^{2}-x^{2}}\right)=-\Re \operatorname{Li}_{1}\left(-\beta e^{-\alpha^{2}-x^{2}}\right) .
$$

To show that the distribution of $N_{c}$ is a Gaussian, we need to know that the integral expression in (18) (for the dominant choice of \pm ) is nonzero. The integral clearly cannot be negative, because it has an interpretation in terms of variance, but a priori it could be zero, in which case the $o(1)$ error term would control the variance, and we would be unable to characterize the distribution of $N_{c}$. Ideally we would like to show that it always positive, but we only do this for $A \leq 1$, or else $A>1$ but $\alpha=0$. Recall that under these conditions $Z_{-}$is dominant.

Lemma 6 If $0<\beta \leq 1$, or else $\beta>1$ but $\alpha=0$, then

$$
-\int_{-\infty}^{\infty} \operatorname{Li}_{-1}\left(-\beta e^{2 i \alpha x-x^{2}}\right) d x>0
$$

Proof: As above, under either of the hypotheses, equation (21) simplifies to

$$
\int_{-\infty}^{\infty} \operatorname{Li}_{-1}\left(-\beta e^{2 i \alpha x-x^{2}}\right) d x=\sqrt{\pi} \operatorname{Li}_{-1 / 2}\left(-\beta e^{-\alpha^{2}}\right)=\int_{-\infty}^{\infty} \operatorname{Li}_{-1}\left(-\beta e^{-\alpha^{2}-x^{2}}\right) d x
$$

but $-\mathrm{Li}_{-1}\left(-\beta e^{-\alpha^{2}-x^{2}}\right)=\beta e^{-\alpha^{2}-x^{2}} /\left(1+\beta e^{-\alpha^{2}-x^{2}}\right)^{2}>0$.

Lemma 7 The curve for $\log Z$ attains its (unique) maximum value when $\alpha=0$.

Proof: When $0<\beta$ we have $\log \left(1+\beta e^{-x^{2}}\right) \geq \Re \log \left(1 \pm \beta e^{2 i \alpha x-x^{2}}\right)$, with strict inequality when $\pm e^{2 i \alpha x} \neq 1$, so $-\int_{-\infty}^{\infty} \operatorname{Li}_{1}\left(\mp \beta e^{2 i \alpha x-x^{2}}\right) d x \leq-\int_{-\infty}^{\infty} \operatorname{Li}_{1}\left(-\beta e^{-x^{2}}\right) d x$, with strict inequality unless both $\alpha=0$ and $\mp=-$.

In contrast, the curve for $\left\langle N_{c}\right\rangle$ does not always attain its maximum value when $\alpha=0$.

\subsection{Asymptotics for large $\alpha$}

In Figure 5 the curves for $\log Z$ and $\left\langle N_{c}\right\rangle$ appear to asymptote out to a positive constant, while the curve for $\sigma^{2}\left(N_{c}\right)$ decays to 0 . The following theorem gives the large- $\alpha$ asymptotics of these curves which are given by the integrals in (16), (17), and (18) in Theorem 2.

Theorem 3 For positive $\beta$ and real $\alpha$,

$$
\begin{aligned}
\lim _{|\alpha| \rightarrow \infty}-\int_{-\infty}^{\infty} \operatorname{Li}_{1}\left( \pm \beta e^{2 i \alpha x-x^{2}}\right) d x & = \begin{cases}\frac{4}{3}(\log \beta)^{3 / 2} & \beta \geq 1 \\
0 & \beta \leq 1\end{cases} \\
\lim _{|\alpha| \rightarrow \infty}-\int_{-\infty}^{\infty} \operatorname{Li}_{0}\left( \pm \beta e^{2 i \alpha x-x^{2}}\right) d x & = \begin{cases}2(\log \beta)^{1 / 2} & \beta \geq 1 \\
0 & \beta \leq 1\end{cases} \\
\lim _{|\alpha| \rightarrow \infty}-\alpha^{2} \int_{-\infty}^{\infty} \operatorname{Li}_{-1}\left( \pm \beta e^{2 i \alpha x-x^{2}}\right) d x & = \begin{cases}(\log \beta)^{1 / 2} & \beta \geq 1 \\
0 & \beta \leq 1\end{cases}
\end{aligned}
$$


Proof: These integrals are evaluated in (20) and (21). When $|\alpha| \rightarrow \infty$, the main terms in (20) and $(21),-\sqrt{\pi} \operatorname{Li}_{\nu+1 / 2}\left( \pm \beta e^{-\alpha^{2}}\right)$ for $\nu=1,0,-1$, become negligible. Thus we only have to compute the "branch-cut terms" when $\beta>1$. For (20) these terms can be approximated for large $\alpha$ as follows. Let $\gamma=\log \beta-\alpha^{2}$. The identity

$$
\sqrt{x+i y}+\sqrt{x-i y}=\sqrt{2\left(x+\sqrt{x^{2}+y^{2}}\right)}
$$

is easily verified by squaring both sides, and allows us to rewrite the sum in (20) as

$$
\sum_{|k|<|\alpha| \sqrt{\log \beta} / \pi} 2 \pi\left(|\alpha|-\sqrt{\left(-\gamma+\sqrt{\gamma^{2}+4 \pi^{2} k^{2}}\right) / 2}\right)
$$

where $k$ is integer for positive $\beta$ (for $Z_{+}$) or half-integer for negative $\beta$ (for $Z_{-}$).

Suppose $|\alpha| \gg \sqrt{\log \beta}$. Then $k$, which ranges up to $(|\alpha| / \pi) \sqrt{\log \beta}$, is much smaller than $|\gamma|=(1+o(1)) \alpha^{2}$, and we have

$$
\begin{aligned}
\sqrt{\left(-\gamma+\sqrt{\gamma^{2}+4 \pi^{2} k^{2}}\right) / 2} & =\sqrt{-\gamma} \sqrt{\left(1+\sqrt{1+4 \pi^{2} k^{2} / \gamma^{2}}\right) / 2} \\
& =\sqrt{-\gamma} \sqrt{1+\left(\pi^{2}+o(1)\right) k^{2} / \gamma^{2}} \\
& =\sqrt{\alpha^{2}-\log \beta}\left(1+\left(\pi^{2} / 2+o(1)\right) k^{2} / \gamma^{2}\right) \\
& =|\alpha|\left(1-(1 / 2+o(1)) \log \beta / \alpha^{2}\right)\left(1+\left(\pi^{2} / 2+o(1)\right) k^{2} / \alpha^{4}\right) \\
& =|\alpha|-\frac{1+o(1)}{2|\alpha|}\left(\log \beta-\pi^{2} k^{2} / \alpha^{2}\right) .
\end{aligned}
$$

In particular

$$
2 \pi\left(|\alpha|-\sqrt{\left(-\gamma+\sqrt{\gamma^{2}+4 \pi^{2} k^{2}}\right) / 2}\right)=\frac{\pi+o(1)}{|\alpha|}\left(\log \beta-\pi^{2} k^{2} / \alpha^{2}\right)
$$

and we sum this over $k$ 's for which the quantity is positive. If $|\alpha| \gg 1 / \sqrt{\log \beta}$ then we can approximate this sum with an integral, and the integral is

$$
\frac{\pi+o(1)}{|\alpha|} \frac{2}{3} \log \beta \times \frac{2|\alpha|}{\pi} \sqrt{\log \beta}=(1+o(1)) \frac{4}{3}(\log \beta)^{3 / 2} .
$$

These are the asymptotics for $\nu=1$. For integer $\nu \leq 0$ the range of $k$ is the same as it is for $\nu=1$, i.e. up to about $|\alpha| \sqrt{\log \beta} / \pi$. Thus we need to evaluate

$$
\sqrt{\pi} \Gamma(1 / 2-\nu) \sum_{|k|<|\alpha| \sqrt{\log \beta} / \pi}\left[\alpha^{2}-\log \beta+2 \pi i k\right]^{-1 / 2+\nu} .
$$

For $\alpha \gg \sqrt{\log \beta}, k \ll \alpha^{2}$ so that

$$
\left[\alpha^{2}-\log \beta+2 \pi i k\right]^{-1 / 2+\nu}+\left[\alpha^{2}-\log \beta-2 \pi i k\right]^{-1 / 2+\nu} \approx 2|\alpha|^{2 \nu-1}
$$

and if $\alpha \gg 1 / \sqrt{\log \beta}$ the summation is approximately an integral which is asymptotically

$$
\frac{2}{\sqrt{\pi}} \Gamma(1 / 2-\nu) \alpha^{2 \nu} \sqrt{\log \beta}
$$

Taking $\nu=0$ and $\nu=-1$ give the desired results for the $\left\langle N_{c}\right\rangle$ and $\sigma^{2}\left(N_{c}\right)$ integrals. 


\subsection{Crossover locations}

If $A \leq 1$ then $Z_{-}$always exceeds $Z_{+}$. But for larger $A$ there are crossover values for $\alpha$ at which $Z_{-}$and $Z_{+}$alternate in significance. Since $Z_{-}$and $Z_{+}$count configurations in different $\mathbb{Z}_{2}$-homology classes (see (4)), we conclude that the crossover values are the places where the typical homology class of a lattice path changes. So there is a phase transition at these points: the topology of a typical configuration changes.

The crossover values $\alpha$ for a fixed $\beta=A^{q}$ satisfy an implicit (and transcendental) equation. Instead of solving these equations directly we can derive an analytic expression for the crossover $\alpha$ 's as a function of $\gamma=\log \beta-\alpha^{2}$, and then given $\gamma$ and the crossover $\alpha$ we can calculate the corresponding $\beta$. In this way we can parametrically plot these critical pairs $(\beta, \alpha)$ as a function of $\gamma$. For example, for the 0th crossover we have

$$
\begin{aligned}
& \epsilon^{1 / 2} q^{3 / 2} Z_{-}+o(1)=-\sqrt{\pi} \operatorname{Li}_{3 / 2}\left(-\beta e^{-\alpha^{2}}\right) \\
& \epsilon^{1 / 2} q^{3 / 2} Z_{+}+o(1)=-\sqrt{\pi} \operatorname{Li}_{3 / 2}\left(\beta e^{-\alpha^{2}}\right)+2 \pi\left(\alpha-\sqrt{\alpha^{2}-\log \beta}\right)
\end{aligned}
$$

from which we can solve

$$
\alpha=\frac{\mathrm{Li}_{3 / 2}\left(e^{\gamma}\right)-\mathrm{Li}_{3 / 2}\left(-e^{\gamma}\right)}{2 \sqrt{\pi}}+\sqrt{-\gamma} .
$$

Similarly, for the $r$ th crossover we have

$$
(-1)^{r} \alpha=\frac{\mathrm{Li}_{3 / 2}\left(e^{\gamma}\right)-\mathrm{Li}_{3 / 2}\left(-e^{\gamma}\right)}{2 \sqrt{\pi}}+\sum_{k=-r}^{r}(-1)^{k} \sqrt{-\gamma+k \pi i} .
$$

Next let us approximate these crossovers for $r$ fixed and $\gamma$ large. For large $\gamma$ we can substitute $\nu=3 / 2$ into the asymptotic series expansions (25) and (26) for $\operatorname{Li}_{\nu}\left( \pm e^{\gamma}\right)$ to write

so that

$$
\begin{gathered}
-\mathrm{Li}_{3 / 2}\left(-e^{\gamma}\right) \approx \frac{4}{3} / \pi^{1 / 2} \gamma^{3 / 2}+\frac{1}{6} \pi^{3 / 2} / \gamma^{1 / 2}+\frac{7}{480} \pi^{7 / 2} / \gamma^{5 / 2}+\cdots \\
\operatorname{Li}_{3 / 2}\left(e^{\gamma}\right) \approx-\frac{4}{3} / \pi^{1 / 2} \gamma^{3 / 2}-2 \sqrt{\pi} i \sqrt{\gamma}+\frac{1}{3} \pi^{3 / 2} / \gamma^{1 / 2}+\frac{1}{60} \pi^{7 / 2} / \gamma^{5 / 2}+\cdots
\end{gathered}
$$

$$
\begin{aligned}
(-1)^{r} \alpha & \approx \frac{\pi}{4} \gamma^{-1 / 2}+\sum_{k=1}^{r}(-1)^{k}[\sqrt{-\gamma+k \pi i}+\sqrt{-\gamma-k \pi i}] \\
& =\frac{\pi}{4} \gamma^{-1 / 2}+\sum_{k=1}^{r}(-1)^{k} \sqrt{2\left(-\gamma+\sqrt{\gamma^{2}+k^{2} \pi^{2}}\right)} \\
& \approx \frac{\pi}{4} \gamma^{-1 / 2}+\sum_{k=1}^{r}(-1)^{k} \sqrt{2\left(-\gamma+\gamma\left(1+k^{2} \pi^{2} /\left(2 \gamma^{2}\right)\right)\right)} \\
& =\frac{\pi}{4} \gamma^{-1 / 2}+\sum_{k=1}^{r}(-1)^{k} \sqrt{k^{2} \pi^{2} / \gamma} \\
& =\frac{\pi}{4} \gamma^{-1 / 2}+\sum_{k=1}^{r}(-1)^{k} k \pi / \gamma^{1 / 2} \\
& =(-1)^{r}(r / 2+1 / 4) \pi \gamma^{-1 / 2} .
\end{aligned}
$$


Since $\alpha$ is small, $\gamma \approx \log \beta$, and so the $r$ th crossover occurs at $\alpha \approx(r / 2+1 / 4) \pi / \sqrt{\log \beta}$.

By comparison the nonanalyticities in the curve for $Z_{-}$occur exactly at $\alpha=\pi / \sqrt{\log \beta}(\mathbb{Z}+$ $1 / 2$ ), and the nonanalyticities in the curve for $Z_{+}$occur exactly at $\alpha=\pi / \sqrt{\log \beta} \mathbb{Z}$.

\section{Open problems}

Our analysis for of the lattice paths at the critical point is geared to regions whose aspect ratio is close to a simple rational number, but we do not know how to treat "irrational domains" which do not have a simple rational approximation. Consider for instance the case $a=1, b=c=1 / 2$ when the side lengths $m$ and $n$ are successive Fibonacci numbers. Then the aspect ratio of the region is very close to the Golden ratio, which is not well approximated by simple rationals. In this case we believe that the partition function $Z$ is $\Theta(1)$ (about 2.1), so that with $\Theta(1)$ probability there are no lattice paths. In the event that there are lattice paths, we believe that they connect up into $\Theta(1)$ loops each of length $\Theta\left(n^{4 / 3}\right)$. We have also found empirically that as one varies the aspect ratio of large regions, the smallest value that the partition function $Z$ takes on is very close to 2 . We do not know how to prove any of these conjectures.

In Figure 3 we plotted $\log Z$ and $\left\langle N_{c}\right\rangle$ versus $n / m$ when $a=1, b=c=1 / 2$. As predicted there are spikes in $\log Z$ and $\left\langle N_{c}\right\rangle$ when the aspect ratio $n / m$ is near a simple rational $p / q$. But there also appear to be flanking secondary spikes in $\left\langle N_{c}\right\rangle$ when $n / m \approx p / q$ but $|n / m-p / q| \gg 1 / \sqrt{n}$. We do not understand this phenomenon.

When $a=1, b=c=1 / 2+\Theta(1 / n)$ and the aspect ratio is nearly a simple rational $p / q$, the partition function and edge density as a function of $\alpha$ are nonanalytic at certain points. We know that the homology type of the strands changes at these nonanalyticities. We conjecture that when $\alpha=0$ each loop winds around exactly $p$ times horizontally and $q$ times vertically. For large enough values of $\alpha$, when one follows a loop for $m p+n q$ steps, one does not return to the starting point. We conjecture that the number of nonanalyticities between $\alpha$ and 0 determines how many strands away one ends up after following a loop for $m p+n q$ steps.

Despite our explicit formulas for $\log Z_{ \pm}$and for the expected value and variance of the number of edges, there are some basic properties about these functions that we have not been able to derive. For example, we conjecture that when our formula for $\log Z_{-}$is nonanalytic, our formula for $\log Z_{+}$gives a strictly larger value, and vice versa, and that the formula for $\sigma^{2}\left(N_{c}\right)$ is strictly positive. We also conjecture that for $A>1$ our formula for $\log Z$ as a function of $\alpha$ always exceeds its limiting value as $\alpha \rightarrow \infty$. These conjectures seem fairly evident from the graphs in Figure 5, but it is not obvious how to prove them. One would also like to determine for fixed $A>1$ the maximum and minimum values given by our formula for $\left\langle N_{c}\right\rangle$, since both of these can be different than the limiting $\alpha \rightarrow \infty$ value.

\section{References}

[1] Harry Bateman and A. Erdélyi et al. Higher Transcendental Functions, volume 1. McGraw-Hill, 1953. Based in part on notes left by Bateman, edited by Erdélyi et al. 
[2] H. W. J. Blöte and H. J. Hilhorst. Roughening transitions and the zero-temperature triangular Ising antiferromagnet. Journal of Physics A, 15(11):L631-L637, 1982.

[3] C. Borgs, J. T. Chayes, and C. King. Meissner phase for a model of oriented flux lines. Journal of Physics A, 28(23):6483-6499, 1995.

[4] Raphaël Cerf and Richard Kenyon. The low-temperature expansion of the Wulff crystal in the 3D Ising model. Communications in Mathematical Physics, 222:147-179, 2001.

[5] Joseph T. Chang and Yuval Peres. Ladder heights, Gaussian random walks and the Riemann zeta function. Annals of Probability, 25(2):787-802, 1997.

[6] Henry Cohn, Richard Kenyon, and James Propp. A variational principle for domino tilings. Journal of the American Mathematical Society, 14(2):297-346, 2001. arXiv:math.CO/0008220.

[7] Louis Comtet. Advanced Combinatorics. D. Reidel Publishing Co., 1974.

[8] Marcel den Nijs. The domain wall theory of two-dimensional commensurateincommensurate phase transitions. In C. Domb and J. L. Lebowitz, editors, Phase Transitions and Critical Phenomena, volume 12, pages 219-333. Academic Press, 1988.

[9] R. B. Dingle. On the Bose-Einstein integrals $\mathcal{B}_{p}(\eta)=(p !)^{-1} \int_{0}^{\infty} \varepsilon^{p}\left(e^{\varepsilon-\eta}-1\right)^{-1} d \varepsilon$. Applied Scientific Research, 6(4):240-244, 1957.

[10] R. B. Dingle. On the Fermi-Dirac integrals $\mathcal{F}_{p}(\eta)=(p !)^{-1} \int_{0}^{\infty} \varepsilon^{p}\left(e^{\varepsilon-\eta}+1\right)^{-1} d \varepsilon$. Applied Scientific Research, 6(4):225-239, 1957.

[11] Michael E. Fisher. Walks, walls, wetting and melting. Journal of Statistical Physics, 34(5-6):667-729, 1984.

[12] Anna Galluccio and Martin Loebl. On the theory of Pfaffian orientations. I. Perfect matchings and permanents. Electronic Journal of Combinatorics, 6(1):\#R6, 1999.

[13] H. Y. Huang, F. Y. Wu, H. Kunz, and D. Kim. Interacting dimers on the honeycomb lattice: An exact solution of the five-vertex model. Physica A, 228(1-4):1-32, 1996. arXiv:cond-mat/9510161.

[14] P. W. Kasteleyn. Graph theory and crystal physics. In Frank Harary, editor, Graph Theory and Theoretical Physics. Academic Press, 1967.

[15] Richard Kenyon. Local statistics of lattice dimers. Annales de l'Institut Henri Poincaré - Probabilités et Statistiques, 33(5):591-618, 1997. arXiv:math.CO/0105054.

[16] Leonard Lewin. Polylogarithms and Associated Functions. North Holland, 1981.

[17] Ernst Lindelöf. Le Calcul des Résidus et ses Applications a la Théorie des Fonctions. Gauthier-Villars, 1905.

[18] W. T. Lu and F. Y. Wu. Dimer statistics on the Möbius strip and the Klein bottle. Physics Letters A, 259(2):108-114, 1999. arXiv:cond-mat/9906154. 
[19] Wentau T. Lu and F. Y. Wu. Close-packed dimers on nonorientable surfaces, 2001. arXiv:cond-mat/0110035.

[20] J. F. Nagle. Theory of biomembrane phase transitions. Journal of Chemical Physics, 58:252-264, 1973.

[21] W. F. Pickard. On polylogarithms. Publicationes Mathematicae, 15(1-4):33-43, 1968.

[22] V. Popkov, Doochul Kim, H. Y. Huang, and F. Y. Wu. Lattice statistics in three dimensions: solution of layered dimer and layered domain wall models. Physical Review E, 56(4):3999-4008, 1997. arXiv:cond-mat/9703065.

[23] M. Prähofer and H. Spohn. An exactly solved model of three-dimensional surface growth in the anisotropic KPZ regime. Journal of Statistical Physics, 88(5-6):999-1012, 1997. arXiv:cond-mat/9612209.

[24] Tullio Regge and Riccardo Zecchina. Combinatorial and topological approach to the 3D Ising model. Journal of Physics A, 33:741-761, 2000. arXiv:cond-mat/9909168.

[25] Scott Sheffield. Gibbs measure uniqueness results for Lipschitz random surfaces, 2001. In preparation.

[26] Serge Tabachnikov. Billiards. Panoramas et Synthèses. Société Mathématique de France, 1995.

[27] Glenn Tesler. Matchings in graphs on non-orientable surfaces. Journal of Combinatorial Theory, Series B, 78:198-231, 2000.

[28] C. Truesdell. On a function which occurs in the theory of the structure of polymers. The Annals of Mathematics, 46(1):144-157, 1945.

[29] F. Y. Wu. Exactly soluable model of the ferroelectric phase transition in two dimensions. Physical Review Letters, 18(15):605-607, 1967.

[30] F. Y. Wu. Remarks on the modified potassium dihydrogen phosphate model of a ferroelectric. Physical Review, 168(2):539-543, 1968.

[31] F. Y. Wu and H. Y. Huang. Exact solution of a vertex model in d dimensions. Letters in Mathematical Physics, 29(3):205-213, 1993.

[32] F. Y. Wu and H. Y. Huang. Exact solution of a lattice model of flux lines in superconductors. Physika A, 205:31-40, 1994.

\section{A Polylogarithms}

The polylogarithm function $\operatorname{Li}_{\nu}(z)$ is defined by

$$
\operatorname{Li}_{\nu}(z)=\sum_{n=1}^{\infty} \frac{z^{n}}{n^{\nu}}
$$


for $|z|<1$ and by analytic continuation elsewhere. We may for instance write $\operatorname{Li}_{1}(z)=$ $-\log (1-z), \operatorname{Li}_{0}(z)=z /(1-z)$, and $\operatorname{Li}_{-1}(z)=z /(1-z)^{2}$. The polylogarithm function has the convenient property that

$$
z \frac{\partial \mathrm{Li}_{\nu}(z)}{\partial z}=\mathrm{Li}_{\nu-1}(z)
$$

Appell's integral expression

$$
\operatorname{Li}_{\nu}(z)=\frac{1}{\Gamma(\nu)} \int_{0}^{\infty} \frac{z s^{\nu-1} d s}{e^{s}-z}
$$

is valid for $\Re(\nu)>0$ and $z \notin[1, \infty)$, and defines the principal branch of the polylogarithm. The polylogarithm has an interesting Riemann surface. When one crosses the branch cut $[1, \infty)$ in the positive direction, the polylogarithm increases by $\frac{2 \pi i}{\Gamma(\nu)}(\log z)^{\nu-1}$. (Both this and the defining series expansion for $\mathrm{Li}_{\nu}$ are readily derived from Appell's integral expression.) For nonpositive integer $\nu$ this quantity is 0 , consistent with the fact that $\mathrm{Li}_{\nu}$ is a rational function for these values of $\nu$. Unless $\nu$ is an integer $\leq 1$, the $\frac{2 \pi i}{\Gamma(\nu)}(\log z)^{\nu-1}$ term creates a second branch point at $z=0$ off the principal branch. The function $\operatorname{Li}_{\nu}(z)$ is analytic in both $z$ and $\nu$, except for a singularity at $z=1$ (and $z=0$ off the principal branch). For further background see Bateman and Erdélyi et al. [1, Chapt. 1 §11], Truesdell [28], Dingle $[10,9]$, and Lewin [16].

When $z$ is on the principal branch and near 1, the series expansion

$$
\operatorname{Li}_{\nu}(z)=\Gamma(1-\nu)(-\log z)^{\nu-1}+\sum_{n=0}^{\infty} \zeta(\nu-n) \frac{(\log z)^{n}}{n !}
$$

was given by Lindelöf [17, pp. 138-141] (derivations are also given in [28] and [5]), and is absolutely convergent when $|\log z|<2 \pi$.

We also use the asymptotic series expansions for $\operatorname{Li}_{\nu}(z)$ when $|z| \rightarrow \infty$. For large positive $x$ these are

$$
\operatorname{Li}_{\nu}(-x)=-\cos (\pi \nu) \operatorname{Li}_{\nu}(-1 / x)+2 \sum_{k=0}^{\infty} \frac{\left(1 / 2^{2 k-1}-1\right) \zeta(2 k)}{\Gamma(\nu+1-2 k)}(\log x)^{\nu-2 k}
$$

and

$$
\operatorname{Li}_{\nu}(x)=-\cos (\pi \nu) \operatorname{Li}_{\nu}(1 / x) \pm \pi i \frac{(\log x)^{\nu-1}}{\Gamma(\nu)}+2 \sum_{k=0}^{\infty} \frac{\zeta(2 k)}{\Gamma(\nu+1-2 k)}(\log x)^{\nu-2 k} .
$$

Aside from the $-\cos (\pi \nu) \operatorname{Li}_{\nu}( \pm 1 / x)$ terms, the asymptotic series expansions (25) and (26) were derived by Sommerfeld and Clunie respectively. Rhodes first derived (25) for the case of integer $\nu$, and for these $\nu$ the expansions (25) and (26) have only finitely many nonzero terms. For noninteger $\nu$ the expansions (25) and (26) diverge, and the $-\cos (\pi \nu) \operatorname{Li}_{\nu}( \pm 1 / x)$ term is dominated by each term in the divergent series expansion, so one may wonder what role it plays. Dingle [10, eqn 17] [9, eqn 11] showed how to make practical computational use of these series by truncating the series after finitely many terms and providing convergent series expansions for the remainder when the $-\cos (\pi \nu) \operatorname{Li}_{\nu}( \pm 1 / x)$ term is present. For positive $x>1$ we are evaluating $\operatorname{Li}_{\nu}(x)$ on the branch cut of the principal branch; the \pm sign is positive when the branch cut is just above the real axis, which is the usual convention. See also Pickard [21, eqn 3.5] for the asymptotics for large complex values of $z$. 


\section{B Notation}

$a, b, c$ : weights of edges in the three different directions. $a$ is weight for vertex not being in a loop, $b$ is weight for horizontal step, and $c$ is weight for vertical step.

$m$ : horizontal length of torus

$n$ : vertical length of torus

Simplifying assumptions: $b<a, b / a=\Theta(1), 1-b / a=\Theta(1), n=\Theta(m)$

$p / q$ : rational approximation of $n b /(m c), \operatorname{gcd}(p, q)=1$. Intuitively $p$ is the number of horizontal windings of loops, and $q$ is the number of vertical windings of loops, but this intuition is not quite accurate if "ratcheting" takes place.

$W$ is a measure of close the rational approximation $p / q \approx n b /(m c)$ needs to be. For fixed $p$ and $q, W=\Theta(1 / \sqrt{n})$. More precisely,

$W=\sqrt{q \epsilon} /(\pi p)=\sqrt{2 q n a b} /(p m(a-b)) \approx \sqrt{2 /(p m c)} \approx \sqrt{2 /(q n b)}$

$\alpha$ is measure of the error in the approximation $p / q \approx n b /(m c)$ on the scale of $W$ :

$\frac{n b q}{m c p} \approx \frac{n b q}{m(a-b) p}=1+\alpha W$.

$A=\left(\frac{c}{a-b}\right)^{n}$

$A$ is a measure of how close the weights $a, b$, and $c$ are to the phase transition $a=b+c$. When $a=b+c, A=1$, and $A$ is sensitive to perturbations on the order of $\Theta(1 / n)$. $A^{q}$ appears in many of our formulas, and is approximately symmetric in the parameters:

$$
\log A^{q} \approx(b+c-a) \sqrt{\frac{m n p q}{b c}}
$$

$\phi=\frac{2 \pi n b}{m(a-b)}$

$\epsilon=\frac{2 \pi^{2} n a b}{m^{2}(a-b)^{2}}$

$z_{k}=-e^{i \theta_{k}}, \theta_{k}=2 \pi k / m ; k \in \mathbb{Z}_{m}+\sigma / 2$ for $Z_{\sigma \tau}$

$r_{k}=(a-b)^{n}\left|a+b z_{k}\right|^{-n}=e^{-\epsilon k^{2}+O\left(k^{4} / m^{3}\right)}$

$\phi_{k}=\arg \left(a+b z_{k}\right)^{-n}=\phi k+O\left(k^{3} / m^{2}\right)$ 